\title{
ROBUSTLY STABLE MULTIESTIMATION SCHEME FOR ADAPTIVE CONTROL AND IDENTIFICATION WITH MODEL REDUCTION ISSUES
}

\author{
A. BILBAO-GUILLERNA, M. DE LA SEN, A. IBEAS, \\ AND S. ALONSO-QUESADA
}

Received 17 May 2004

\begin{abstract}
A discrete pole-placement-based and multiestimation-based adaptive control scheme involving a relative adaptation dead zone is presented for a plant with known poles and unknown zeros. The basic usefulness of the proposed multiestimation scheme is related to the use of a set of models of reduced order associated with the multiestimation scheme instead of a high-order one. Depending on the frequency spectrum characteristics of the input and on the estimates evolution, the multiestimation scheme selects on-line the most appropriate model and its related estimation scheme in order to improve the identification and control performances. Robust closed-loop stability is proved even in the presence of unmodeled dynamics of sufficiently small sizes as it has been confirmed by simulation results. The scheme chooses in real time the estimator/controller associated with a particular reduced model possessing the best performance according to an identification performance index by implementing a switching rule between estimators. The switching rule is subject to a minimum residence time at each identifier/adaptive controller parameterization for closed-loop stabilization purposes. A conceptually simple higher-level supervisor, based on heuristic updating rules which estimate on-line the weights of the switching rule between estimation schemes, is discussed.
\end{abstract}

\section{Introduction}

The challenge of control theory nowadays is to develop control system schemes able to achieve a good performance in terms of speed, accuracy, and stability for increasingly complex systems, including the presence of large uncertainties in the controlled system. The dynamics of almost all real systems is nonlinear with multiple equilibrium points so that sometimes their behavior may change abruptly according to the operation conditions $[7,23]$. These operation conditions depend on the magnitude and type of the input signal applied to the system, particularly on the frequency range at which the input signal belongs. As a consequence, in many industrial applications, it is not reasonable to assume that the same plant model remains adequate as time progresses. One of the fields where the model order used is more relevant related to the dominant frequencies of the applied input signal is the design of controlled systems such as robots and space structures with 
structural flexibility [21]. In such a type of problems, the selection and placement of sensors and actuators is an important design step since the sensor data may be redundant or not depending on whether they are placed to measure exactly the same parameter [10]. That selection and placement must be optimized according to, for instance, the key points in the flexible structure shape to measure the relevant modes. This paper deals with the problem of controlling a plant with known poles and unknown zeros by using an appropriate reduced-order plant model. The main theoretical idea relies on decomposing the plant transfer function as a sum of several elementary fractions of different orders, in general, with unknown numerators. In this way, a set of plant nominal models of reduced order, with unknown numerator polynomials, are considered by selecting combinations of such fractions to represent different models. An identifier/adaptive controller pair is used for each of those unknown models of known denominator polynomials. Furthermore, a switching rule between the various identifier/adaptive controller pairs, based on the identification quality of each one, is used to choose on-line the most appropriate model at each time interval depending on the transient behavior and reference input spectrum characteristics $[6,11,13,15,17,18,19,20,22]$. The identification procedure basically consists in estimating the numerator polynomial coefficients of the reduced-order models within the multiestimation scheme. A higher-level supervisor is used with the aim of selecting on-line the best value of a weighting factor. In many practical applications, it is crucial to elucidate either the number of dominant modes to be considered for identification purposes or the method to be used in the identification process as for instance in [5] where three different identification methods were proposed, namely:

(i) the so-called empirical transfer function estimation (ETFE),

(ii) a modification of the above method consisting in calculating the average cross spectrum between the input and output both divided by the input spectrum,

(iii) a truncated Fourier transform based on the estimation method.

The obtained identification performance was found to be very different from one method to another depending on the input frequency. This feature is an empirical proof of the importance of the selection of the identification method and, as a result, the high dependence on the identification performance of the model and its order and relative order is also foreseen. In this paper, we give a method to integrate the on-line model order choice with the reference input spectrum in adaptive control. The mechanism is to implement a switching rule between several estimators of different orders prior to the adaptive controller parameterization. The overall process is stated as an automatic task that does not require any on-line designer operation. The time intervals between consecutive switches are subject to a minimum residence time that guarantees the closed-loop stability and acceptable transient behavior. The main idea behind the proposed scheme is that the reduction model techniques may be addressed and linked with multiestimation techniques while taking into account the transient response generated from each particular reference input used. This is the main novelty in this paper with respect to previous results concerned with multiestimation (see, e.g., $[6,11,13,15,17,18,19,20,22]$ ) where the idea of model reduction was not integrated with that of multiestimation.

The paper is organized as follows. Section 2 deals with the system to be controlled as well as with the multiestimation and adaptive controller architecture together with 
the basic assumptions needed for stability and convergence purposes. Also, a higher-level supervisor for the weights of the identification performance indexes is discussed while the switchings between the estimators within the parallel multiestimation scheme are considered as a lower-level supervisor. The above higher-level supervisor is designed based on empirical rules to improve the tracking performance by updating on-line the weights of the identification performance indexes. In Section 3, the main properties about identification algorithms, control law, and closed-loop stability are given. In Section 4, some computer simulations and their corresponding discussion are presented. Section 5 discusses the identification of the different reduced-order models for nonlinear plants including some numerical examples. Finally, conclusions end the paper.

\section{Problem statement}

2.1. Plant description. Consider the linear and time-invariant discrete SISO plant,

$$
A\left(q^{-1}\right) y_{k}=B\left(q^{-1}\right) u_{k}
$$

where $u_{k}$ and $y_{k}$ are the input and output sequences, respectively, $q^{-1}$ is the one-step delay operator, and the degrees of polynomials, $n=\operatorname{deg}\left(A\left(q^{-1}\right)\right), m=\operatorname{deg}\left(B^{*}(q)\right)$, where $B^{*}(q)=q^{n} B\left(q^{-1}\right)$, are at least $n \geq 1$ and $m \geq 0(n>m)$, respectively.

Assumption 2.1. All the plant poles and their multiplicities are known.

Furthermore, the polynomial $A$ can be written as

$$
A(z)=A_{r}(z) A_{c}(z)
$$

where $A_{r}(z)=\prod_{i=1}^{n_{r}^{\prime}}\left[z-p_{i}\right]^{\partial_{i}}$ is a polynomial containing all real $n_{r}^{\prime}$ distinct poles with respective multiplicities $\partial_{i}\left(1 \leq i \leq n_{r}^{\prime}\right)$, and $A_{c}(z)=\prod_{i=n_{r}^{\prime}+1}^{n_{r}^{\prime}+n_{c}^{\prime} / 2}\left[z^{2}-\left(p_{i}+\bar{p}_{i}\right) z+p_{i} \bar{p}_{i}\right]^{\partial_{i}}$ is a polynomial containing $n_{c}^{\prime} / 2$ distinct pairs of complex conjugate poles with multiplicities $\partial_{i}\left(n_{r}^{\prime}<i \leq n_{r}^{\prime}+n_{c}^{\prime} / 2\right)$ accounting for $n_{r}=\operatorname{deg}\left(A_{r}\right)=\sum_{i=1}^{n_{r}^{\prime}} \partial_{i} ; n_{c}=n-n_{r}=\operatorname{deg}\left(A_{c}\right)=$ $2 \sum_{i=n_{r}^{\prime}+1}^{n_{r}^{\prime}+n_{c}^{\prime} / 2} \partial_{i}$, with $n_{r}$ and $n_{c} / 2$ being the number of total real poles and pairs of complex conjugate poles, respectively. If $\partial_{i}=1$ for all $1 \leq i \leq n_{r}^{\prime}+n_{c}^{\prime} / 2$, then $n_{r}=n_{r}^{\prime}$ and $n_{c}=n_{c}^{\prime}$. Then, factorize $B(z) / A(z)$ such that there are $\partial_{i}$ parallel filters of orders running from unity to $\partial_{i}$ with a scalar gain in the numerator for each simple pole $p_{i}$ of multiplicity $\partial_{i}$ resulting in the decompositions (see Figure 2.1a)

$$
\frac{k_{i, 1}}{z-p_{i}}+\frac{k_{i, 2}}{\left(z-p_{i}\right)^{2}}+\cdots+\frac{k_{i, \partial_{i}}}{\left(z-p_{i}\right)^{\partial_{i}}} \quad\left(i=1,2, \ldots, n_{r}^{\prime}\right) .
$$

For each pair of complex conjugate poles of multiplicity $\partial_{i}$, there are $\partial_{i}$ parallel filters in the decomposition of orders from two to $2 \partial_{i}$ with just one different zero in all of them 
34 Multiestimation for adaptive control and reduced models

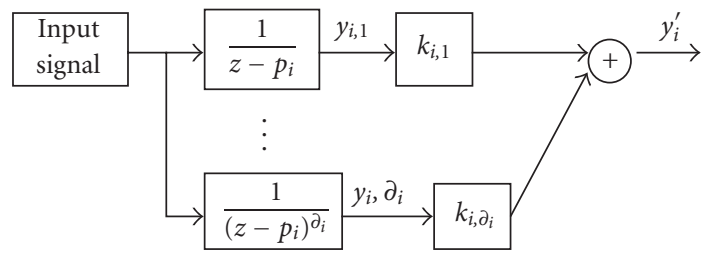

(a)

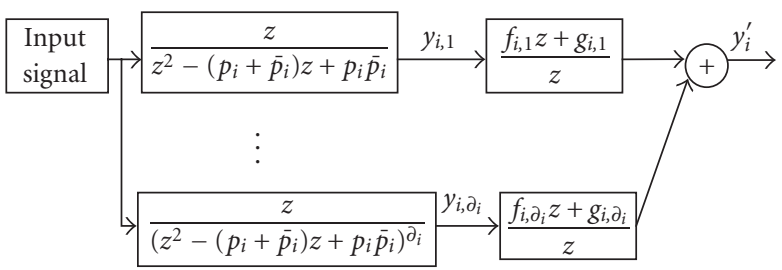

(b)

Figure 2.1. (a) Decomposition for a real pole of multiplicity $\partial_{i}$. (b) Decomposition for a pair of complex conjugate poles with multiplicity $\partial_{i}$.

which results in the decompositions (see Figure 2.1b)

$$
\begin{array}{r}
\frac{f_{i, 1} z+g_{i, 1}}{z^{2}-\left(p_{i}+\bar{p}_{i}\right) z+p_{i} \bar{p}_{i}}+\frac{f_{i, 2} z+g_{i, 2}}{\left(z^{2}-\left(p_{i}+\bar{p}_{i}\right) z+p_{i} \bar{p}_{i}\right)^{2}}+\cdots+\frac{f_{i, \partial_{i} z+g_{i, \partial_{i}}}}{\left(z^{2}-\left(p_{i}+\bar{p}_{i}\right) z+p_{i} \bar{p}_{i}\right)^{\partial_{i}}} \\
\left(i=n_{r}^{\prime}+1, \ldots, n_{r}^{\prime}+\frac{n_{c}^{\prime}}{2}\right) .
\end{array}
$$

Thus, $B(z) / A(z)$ is equivalent to

$$
\frac{B(z)}{A(z)}=\sum_{i=1}^{n_{r}^{\prime}} \sum_{j=1}^{\partial_{i}} \frac{k_{i, j}}{\left(z-p_{i}\right)^{j}}+\sum_{i=n_{r}^{\prime}+1}^{n_{r}^{\prime}+n_{c}^{\prime} / 2} \sum_{j=1}^{\partial_{i}} \frac{f_{i, j} z+g_{i, j}}{\left(z^{2}-\left(p_{i}+\bar{p}_{i}\right) z+p_{i} \bar{p}_{i}\right)^{j}} .
$$

In Figure 2.2, the $B_{i}$ blocks represent the proposed decompositions for each different real pole or each different pair of complex conjugate poles. In this way, the sum of all the $y_{i}^{\prime}$, for $i \in\left\{1,2, \ldots, n_{r}^{\prime}+n_{c}^{\prime} / 2\right\}$, gives us the plant output $y=\sum_{i=1}^{n_{r}^{\prime}+n_{c}^{\prime} / 2} y_{i}^{\prime}$.

Note that (2.1) may be rewritten [14] as

$$
A^{(\ell)}\left(q^{-1}\right) y_{k}=B^{(\ell)}\left(q^{-1}\right) u_{k}+\eta_{k}^{(\ell)},
$$




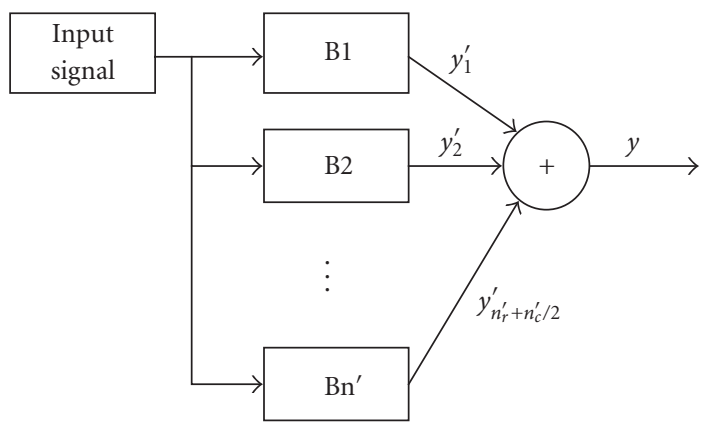

Figure 2.2. Plant decomposition into as many blocks as it has different poles.

where

$$
\frac{B^{(\ell)}(z)}{A^{(\ell)}(z)}=\sum_{i \in N_{r}^{(\ell)}} \sum_{j=1}^{\partial_{i}} \frac{k_{i, j}^{(\ell)}}{\left(z-p_{i}\right)^{j}}+\sum_{i \in N_{c}^{(\ell)}} \sum_{j=1}^{\partial_{i}} \frac{f_{i, j}^{(\ell)} z+g_{i, j}^{(\ell)}}{\left(z^{2}-\left(p_{i}+\bar{p}_{i}\right) z+p_{i} \bar{p}_{i}\right)^{j}}
$$

and $\ell \in N_{e}=\left\{1,2, \ldots, n_{e}\right\}$ ( $n_{e}$ number of reduced-order models). Each element of $N_{e}$ represents a different reduced-order model of the plant, with

(1) $N_{r}^{(\ell)} \subseteq N_{r}=\left\{1,2, \ldots, n_{r}^{\prime}\right\}$; indexing set of $n_{r}^{(\ell)}$ distinct real poles of the $\ell$ th model;

(2) $N_{c}^{(\ell)} \subseteq N_{c}=\left\{n_{r}^{\prime}+1, \ldots, n_{r}^{\prime}+n_{c}^{\prime} / 2\right\}$; indexing set of $n_{c}^{(\ell)}$ distinct pairs of complex conjugate poles of the $\ell$ th model, where either $N_{r}^{(\ell)}$ or $N_{c}^{(\ell)}$ (but not both) may be empty;

(3) $\operatorname{card}\left(N_{r}^{(\ell)}\right)=n_{r}^{(\ell)} \leq n_{r}^{\prime}$; that is, the number of distinct real poles of the $\ell$ th estimation model;

(4) $\operatorname{card}\left(N_{c}^{(\ell)}\right)=n_{c}^{(\ell)} \leq n_{c}^{\prime} / 2$; that is, the number of distinct pairs of complex conjugate poles of the $\ell$ th reduced-order model.

$\eta_{k}^{(\ell)}$ is the total contribution of the unmodeled dynamics to the output in the $\ell$ th model. By using distinct combinations of elements for $N_{r}^{(\ell)}$ and $N_{c}^{(\ell)}$, the number of possible plant models can be calculated as

$$
1 \leq n_{e} \leq \sum_{\ell=1}^{n^{\prime}}\left(\begin{array}{l}
n^{\prime} \\
\ell
\end{array}\right)=\sum_{\ell=1}^{n^{\prime}} \frac{n^{\prime} !}{\ell !\left(n^{\prime}-\ell\right) !}=2^{n^{\prime}}-1 \quad \text { with } n^{\prime}=n_{r}^{\prime}+\frac{n_{c}^{\prime}}{2}
$$

Each reduced plant model could be seen as being a different combination of distinct order filters $B_{(\cdot)}$ (see Figures 2.1 and 2.2). The main idea becomes apparent since any of those filters could be removed because its contribution to the output is sufficiently small compared with that of the rest of filters. However, the decision about which filters are suitable for being deleted from the overall scheme is not always easy, since this depends not only on the plant parameters but also on the importance of the frequency of the reference 
input. This fact can be highlighted by taking just a single block with a real pole of multiplicity unity $\left(F_{i, 1}\right)$, then we can calculate its frequency-dependent gain from the substitution $z=e^{j \omega_{\text {ref }} T_{s}}$ in the modulus of the filter, where $\omega_{\text {ref }}$ is the frequency of the reference input and $T_{s}$ is the sampling period. This makes evident the importance of the input spectrum which may be taken into account, if available, from a priori knowledge:

$$
\text { gain of } F_{i, 1}=\frac{\left|k_{i, 1}\right|}{\sqrt{1+p_{i}^{2}-2 p_{i} \cos \left(w_{\text {ref }} T_{s}\right)}} \in\left[\frac{\left|k_{i, 1}\right|}{1+p_{i}}, \frac{\left|k_{i, 1}\right|}{1-p_{i}}\right] \text {. }
$$

The following standard assumptions are made.

Assumptions 2.2. (1) It is assumed that polynomial degrees $n$ and $m$ are known, and that $A(z)$ and $A^{(\ell)}(z)$ are monic.

(2) All the unstable plant zeros (if any) are known and are also zeros of the reference model.

(3) The reference model $H_{m}(z)=B_{m}(z) / A_{m}(z)$ is exponentially stable, that is, all the zeros of $A_{m}(z)$, which is a monic polynomial, satisfy $|z| \leq 1-\delta$ for some $\delta \in(0,1]$.

(4) There exists a known convex and compact subset $D^{(\ell)} \subseteq \mathbf{D} \subseteq \mathfrak{R}^{2 n}$, for $\ell \in N_{e}$, of the parameter space containing the real nominal plant parameter vectors, so that for all plant parameterization in $D^{(\ell)}$ the polynomials $A^{(\ell)}$ and $B^{(\ell)}$ are coprime for all $\ell \in N_{\ell}$.

(5) Now it is assumed that $N_{e}$ is split into two subsets; namely, $N_{e s}$ and $N_{e u}$, where $N_{e s}$ possesses all the reduced-order models with stable unmodeled dynamics and $N_{e u}$ contains the reduced models with unstable unmodeled dynamics with $N_{e}=N_{e s} \cup N_{e u}$, where $\operatorname{card}\left(N_{e s}\right) \geq 1$, that is, it is not empty.

(6) There exist real known constants $\sigma^{(\ell)} \in(0,1), \alpha_{0}^{(\ell)} \geq 0$, and $\alpha_{1}^{(\ell)} \geq 0$ such that

$$
\left|\eta_{k}^{(\ell)}\right| \leq \bar{\eta}_{k}^{(\ell)}=\alpha_{1}^{(\ell)} \bar{\rho}_{k}^{(\ell)}+\alpha_{0}^{(\ell)}, \quad \text { where } \bar{\rho}_{k}^{(\ell)}=\operatorname{Sup}_{0 \leq j \leq k}\left(\sigma^{(\ell)^{k-j}}\left\|\varphi_{j}^{(\ell)}\right\|\right) \forall \ell \in N_{e s}
$$

If the transfer function from $u_{k}$ to $\eta_{k}^{(\ell)}$ is strictly proper, then the last term in accounting for the supreme in the above formula for $\bar{\rho}_{k}^{(\ell)}$ could be removed resulting in $\bar{\rho}_{k}^{(\ell)}=\operatorname{Sup}_{0 \leq j \leq k-1}\left(\sigma^{(\ell)^{k-j}}\left\|\varphi_{j}^{(\ell)}\right\|\right) \leq \sigma^{(\ell)} \operatorname{Sup}_{0 \leq j \leq k-1}\left(\left\|\varphi_{j}^{(\ell)}\right\|\right)$.

Moreover, $\left|\eta_{k}^{(\ell)}\right| \leq \alpha_{1} \rho_{k}+\alpha_{0}$, where $\rho_{k}=\operatorname{Sup}_{0 \leq j \leq k}\left(\sigma^{k-j}\left\|\varphi_{j}\right\|\right)$ with $\alpha_{1}=\operatorname{Max}\left(\alpha_{1}^{(\ell)}, \ell \in\right.$ $\left.N_{e}\right), \alpha_{0}=\operatorname{Max}\left(\alpha_{0}^{(\ell)}, \ell \in N_{e}\right), \sigma=\operatorname{Max}\left(\sigma^{(\ell)}, \ell \in N_{e}\right)$, and $\left\|\varphi_{j}\right\|=\operatorname{Max}\left(\left\|\varphi_{j}^{(\ell)}\right\|, \ell \in N_{e}\right)$.

The previous assumption may be extended for all $\ell \in N_{u s}$ by making $\sigma^{(\ell)}>1$. Note that the polynomial $B(z)$ of the plant zeros can be expressed uniquely as $B=B^{+} B^{-}$, where $B^{+}$ is monic and $B^{-}$contains all zeros of $B$ satisfying $|z|>1-\delta$ for some $\delta \in(0,1]$ (in particular, $B^{-}$includes all unstable roots of $B$ ). Assumption 2.1 (2.6) implies that the plant is controllable. It will be then used to project the estimates of $A$ and $B$ in $\mathbf{D}$ so as to ensure the controllability of all the estimation models for all time. Assumption 2.1 (2.9) implies that the contribution of the unmodeled dynamics to the output in all the estimates grows 
nonlinearly faster than linearly with the supreme of the total regressor. Moreover, that assumption will then be crucial to prove the closed-loop stability of the adaptive system.

2.2. Parallel multiestimation scheme. A parallel multiestimation scheme is proposed based on the various uncertain plant models (2.7) for the case when the zeros in plant (2.1) are unknown. Each estimation scheme possesses a relative adaptation dead zone so as to freeze the adaptation when the absolute value of identification error is sufficiently small related to a known upper bound of the absolute value of the contribution of the unmodeled dynamics. At the same time, each estimator tentatively parameterizes separately the adaptive controller at all times based on a specific ad hoc diophantine equation based on each nominal part of the various plant estimation models. The main idea behind this philosophy is to switch at appropriate sampling instants between the various plant estimation models so as to appropriately reparameterize the pole-placement-based adaptive controller. Such a strategy will potentially allow the designer to deal with larger or smaller amounts of unmodeled dynamics. The closed-loop stability is guaranteed if the time interval between consecutive switches exceeds a minimum residence time. To simplify the exposition, a parallel multiestimation scheme with a set of only $n_{e}$ estimators of unity relative degree is used based on the plant models (2.7) as follows:

$$
\hat{y}_{k}^{(\ell)}=-\hat{A}_{k}^{(\ell)} y_{k}+\hat{B}_{k}^{(\ell)} u_{k}=\hat{\theta}_{k}^{(\ell)^{T}} \varphi_{k}^{(\ell)} \Longleftrightarrow \hat{A}_{k}^{(\ell)}\left(q^{-1}\right) y_{k}=\hat{B}_{k}^{(\ell)}\left(q^{-1}\right) u_{k}+e_{k}^{(\ell)}
$$

for $\ell=1,2, \ldots, n_{e}$ and all $k \geq 0$, where $\hat{A}_{k}^{(\ell)}\left(q^{-1}\right)=1+\hat{A}_{k}^{(\ell)}\left(q^{-1}\right)$ and $\hat{B}_{k}^{(\ell)}\left(q^{-1}\right)$ are the estimates of the polynomials $A^{(\ell)}\left(q^{-1}\right)$ and $B^{(\ell)}\left(q^{-1}\right)$, respectively, and $e_{k}^{(\ell)}$ is the $\ell$ th identification error for the $k$ th sample which is given by

$$
e_{k}^{(\ell)}=y_{k}-\hat{y}_{k}^{(\ell)}=\hat{A}_{k}^{(\ell)} y_{k}-\hat{B}_{k}^{(\ell)} u_{k}=y_{k}-\hat{\theta}_{k}^{(\ell)^{T}} \varphi_{k}^{(\ell)}=\tilde{\theta}_{k}^{(\ell)^{T}} \varphi_{k}^{(\ell)}+\eta_{k}^{(\ell)}
$$

where $\hat{y}_{k}^{(\ell)}$ is the $\ell$ th estimation of the output for the $k$ th sample. $\hat{\theta}_{k}^{(\ell)}$ and $\varphi_{k}^{(\ell)}$ are, respectively, the estimation of the nominal plant parameter vector and associated regressor defined by

(a) $\hat{\theta}_{\ell}=\left(\hat{\theta}_{r}^{(\ell)^{T}}, \hat{\theta}_{c}^{(\ell)^{T}}\right)^{T}$, where $\theta_{r}^{(\ell)}$ and $\theta_{c}^{(\ell)}$ are the parameterizations related to real poles and the pair of complex conjugate poles, respectively;

(b) $\hat{\theta}_{r}^{(\ell)}=\left(\hat{\theta}_{N_{r}^{(\ell)}(1)}^{(\ell)^{T}}, \ldots, \hat{\theta}_{N_{r}^{(\ell)}\left(n_{r}^{(\ell)}\right)}^{(\ell)}\right)^{T}$ and $\hat{\theta}_{c}^{(\ell)}=\left(\hat{\theta}_{N_{c}^{\prime}(\ell)}^{(\ell)}(1), \ldots, \hat{\theta}_{N_{c}^{(\ell)}\left(n_{c}^{(\ell)}\right)^{T}}^{(\ell)}\right)^{T}$.

Each $N_{r}^{(\ell)}(j)$ for $1 \leq j \leq n_{r}^{(\ell)}$ is one of the elements of $N_{r}$ depending on the $\ell$ th model. In the same way, each $N_{c}^{(\ell)}(j)$ for $1 \leq j \leq n_{c}^{(\ell)}$ is one of the elements of $N_{c}$. The elements of $\hat{\theta}_{r}^{(\ell)}$ and $\hat{\theta}_{c}^{(\ell)}$ are defined by

$$
\begin{aligned}
& \hat{\theta}_{i}^{\prime(\ell)}=\left(\hat{k}_{i, 1}^{(\ell)}, \ldots, \hat{k}_{i, \partial_{i}}^{(\ell)}\right)^{T} \quad \text { if } i \in N_{r} ; \\
& \hat{\theta}_{i}^{\prime(\ell)}=\left(\hat{f}_{i, 1}^{(\ell)}, \hat{g}_{i, 1}^{(\ell)}, \ldots, \hat{f}_{i, \partial_{i}}^{(\ell)}, \hat{g}_{i, \partial_{i}}^{(\ell)}\right)^{T} \quad \text { if } i \in N_{c} .
\end{aligned}
$$




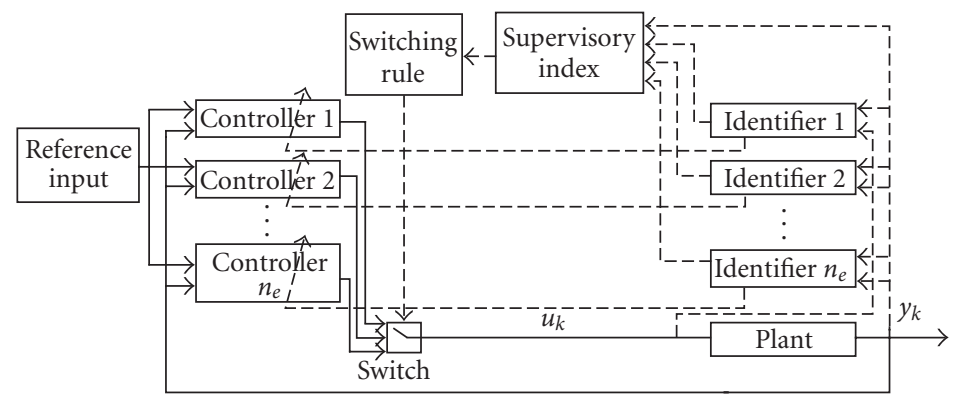

Figure 2.3. Multiestimation scheme.

The associated regressor is given by $\varphi_{k}^{(\ell)}=\left(\varphi_{r, k}^{(\ell)^{T}}, \varphi_{c, k}^{(\ell)^{T}}\right)^{T}$ for $1 \leq \ell \leq n_{e}$ and $k \geq 0$, where $\varphi_{r, k}^{(\ell)}=\left(\varphi_{N_{r}^{(\ell)}(1), k}^{T}, \ldots, \varphi_{N_{r}^{(\ell)}\left(n_{r}^{(\ell)}\right), k}^{T}\right)^{T}$ and $\varphi_{c, k}^{(\ell)}=\left(\varphi_{N_{c}^{(\ell)}(1), k}^{T}, \ldots, \varphi_{N_{c}^{(\ell)}\left(n_{c}^{(\ell)}\right), k}^{T}\right)^{T}$, where $\varphi_{i, k}^{\prime}=$ $\left(y_{i, 1, k}, \ldots, y_{i, \partial_{i}, k}\right)^{T}$ if $i \in N_{r}$ and $\varphi_{i, k}^{\prime}=\left(y_{i, 1, k}, y_{i, 1, k-1}, \ldots, y_{i, \partial_{i}, k}, y_{i, \partial_{i}, k-1}\right)^{T}$ if $i \in N_{c}$. Figure 2.1 shows how the elements of $\varphi_{i, k}^{\prime}$ are calculated. It is usual to know a compact subset of the parameter space $\mathbf{D} \subseteq \mathfrak{R}^{2 n}$ where the nominal real plant parameter vector belongs. This knowledge allows the designer the use of projections of the estimates within such a domain. If the estimation algorithm starts running with a nominal estimated vector being far from the real plant parameter vector, then the transient will have large deviations from the desired output resulting in an unsuitable performance. In this work we have chosen a parallel multiestimation scheme to improve the transient response of the adaptive system. The architecture of the multiestimation scheme is represented in Figure 2.3.

There exist $n_{e}$ estimation algorithms running in parallel (i.e., at each sampling time $t_{k}$ every algorithm gives the estimated parameter vector $\hat{\theta}_{k}^{(\ell)}$ and the estimated plant output $\hat{y}_{k}^{(\ell)}, \ell \in N_{e}$, based on past plant input and output measurements). Algorithms are different from each other in what is concerned with the estimated parameter vector initialization and/or the kind of the estimation algorithm and integrates the so-called multiestimation scheme. There also exist $n_{e}$ adaptive controller parameterizations (with only one being in operation at each time) such that the $\ell$ th adaptive controller is parameterized at every sampling instant by the $\ell$ th estimation algorithm. Thus, every pair identification algorithm-adaptive controller is indexed with only one integer $\ell \in N_{e}$. Denote by $c_{k}$ the integer in $N_{e}$ that defines the controller (parameterized by its respective identification algorithm) which is active (i.e., connected to the plant for control purposes) at the current time. A switching rule based on the identification errors $e_{k}^{(\ell)}=y_{k}-\hat{y}_{k}^{(\ell)}=$ $\varphi_{k}^{T} \tilde{\theta}_{k}^{(\ell)}+\eta_{k}^{(\ell)}\left(\ell \in N_{e}\right)$ of the $n_{e}$ estimation algorithms chooses at each sampling time $t_{k}=k T_{s}$ the individual estimation scheme which parameterizes the controller at time $t_{k}$ which is in fact connected in feedback to the plant.

Remarks 2.3. (1) At each time, only one parameterization of adaptive controller obtained from one of the estimates of the parallel multiestimation scheme is in operation generating the control input. 
(2) All the estimation algorithms are always running in parallel to calculate all the estimated plant outputs. Also, each respective adaptive controller is updated for all times although only the $\ell$ th controller is generating the plant input.

2.3. Estimation algorithm. The proposed parameter-adaptive algorithm with relative adaptation dead zone is

$$
\begin{aligned}
& \hat{\theta}_{k+1}^{(\ell)}=\hat{\theta}_{k}^{(\ell)}+\frac{s_{k}^{(\ell)} P_{k}^{(\ell)} \varphi_{k}^{(\ell)} e_{k}^{(\ell)}}{1+\varphi_{k}^{(\ell)^{T}} P_{k}^{(\ell)} \varphi_{k}^{(\ell)}} ; \\
& P_{k+1}^{(\ell)}=P_{k}^{(\ell)}-\frac{s_{k}^{(\ell)} P_{k}^{(\ell)} \varphi_{k}^{(\ell)} \varphi_{k}^{(\ell)^{T}} P_{k}^{(\ell)}}{1+\varphi_{k}^{(\ell)^{T}} P_{k}^{(\ell)} \varphi_{k}^{(\ell)}}, \quad P_{0}^{(i)}=P_{0}^{(i)^{T}}>0 ; \\
& s_{k}^{(\ell)}= \begin{cases}0 & \text { if }\left|e_{k}^{(\ell)}\right| \leq \mu^{(\ell)} \bar{\eta}_{k}^{(\ell)}, \\
1-\mu^{(\ell)} \mid \frac{\bar{\eta}_{k}^{(\ell)} \mid}{e_{k}^{(\ell)} \mid} \text { otherwise, }\end{cases}
\end{aligned}
$$

for some real design parameters $\mu^{(\ell)}>1$, all $\ell \in N_{e}$, and all integers $k \geq 0$. The relative dead zone (2.14b) freezes the parametrical and covariance matrix adaptation in (2.14a) if the identification error is sufficiently small related to the available upper-bound function of the contribution of the unmodeled dynamics. This is the basic mechanism which supplies attractive properties of the estimates and allows the stabilization of the closed-loop system in the presence of unmodeled dynamics satisfying Assumptions 2.2 (2.9). Those features are discussed in Section 3.

2.4. Basic adaptive controller. The transfer function of the reference model is $H_{m}(z)=$ $B^{-}(z) B_{m}^{\prime}(z) A_{0}^{(\ell)}(z) / A_{m}(z) A_{0}^{(\ell)}(z)$, where $B_{m}^{\prime}(z)$ contains the free-design reference model zeros, $B^{-}(z)$ is formed by the unstable (assumed known) plant zeros, and $A_{0}^{(\ell)}(z)$ are closed-loop stable pole-zero cancelations which are introduced when necessary to guarantee that the synthesized controller is causal. All the controllers are based on poleplacement (see, e.g., [2]), whose basic scheme is displayed in Figure 2.4. Then, we will consider for each controller the polynomials $R_{k}^{(\ell)}, S_{k}^{(\ell)}$, and $T^{(\ell)}\left(T^{(\ell)}\right.$ depends only on the reference model zeros polynomial which is of constant coefficients), where $T^{(\ell)}=B_{m}^{\prime} A_{0}^{(\ell)}$ and $R_{k}^{(\ell)}$ (monic), $S_{k}^{(\ell)}$ are the unique solutions with degrees fulfilling

$$
\operatorname{deg}\left(R_{k}^{(\ell)}\right)=2 n-i, \quad \operatorname{deg}\left(S_{k}^{(\ell)}\right)=i-1, \quad \operatorname{deg}\left(A_{m} A_{0}^{(\ell)}\right)=2 n
$$

of the polynomial diophantine equation

$$
\hat{A}_{k}^{(\ell)} R_{k}^{(\ell)}+\hat{B}_{k}^{(\ell)} S_{k}^{(\ell)}=A_{m} A_{0}^{(\ell)} \Longleftrightarrow \hat{A}_{k}^{(\ell)} R_{1 k}^{(\ell)}+B^{-} S_{k}^{(\ell)}=A_{m} A_{0}^{(\ell)}
$$

with $R_{k}^{(i)}=\hat{B}_{k}^{(\ell)^{+}} R_{1 k}^{(\ell)}$; since $B^{-}(z)$ is known and $R_{k}, R_{1 k}, B^{+}$, and $\hat{B}_{k}^{(\ell)^{+}}$are monic, $\operatorname{deg}\left(\hat{A}_{k}^{(\ell)}\right)$ $=i$ and $\operatorname{deg}\left(\hat{B}_{k}^{(\ell)}\right)=i-1$, for all $\ell \in N_{e}$ at every sampling instant if the relative degree of the $\ell$ th estimation model is unity. Assumption 2.2(4) is extended in a natural way 


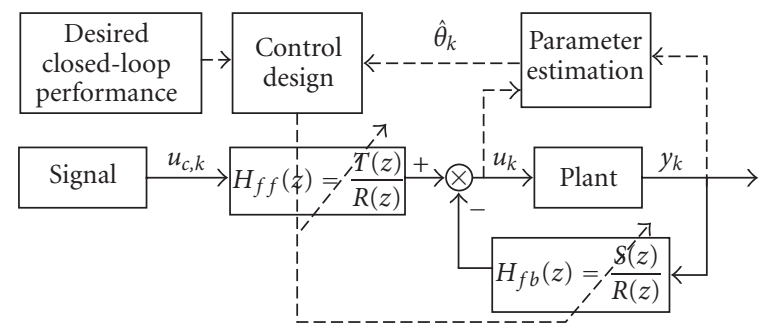

Figure 2.4. Basic adaptive controller.

to the multiestimation scheme by using a posteriori projection of the estimates when necessary as follows.

Assumption 2.4. It is assumed that $\hat{\theta}_{k}^{(\ell)} \in D^{(\ell)}$, then $\left(\hat{A}_{k}^{(\ell)}, \hat{B}_{k}^{(\ell)}\right)$ is a coprime pair over $D^{(\ell)}$ for all $k \geq 0$ and $\ell \in N_{e}$; that is, all the estimation schemes are controllable for all time.

The above assumption would then be useful provided that each pair estimation scheme-adaptive controller parameterization is associated with a different plant operation point. This is often the case, for instance, of some chemical engineering processes. Now it is necessary to elucidate how to choose the current adaptive controller (or, in other words, which active controller at each sampling instant) from the family of parallel controller parameterizations such that the adaptation transients are acceptable in practice while the closed-loop scheme is maintained globally stable. Thus, the basic poleplacement adaptive controller (see Figure 2.4) is reparameterized by one of the estimators of the multiestimation scheme during appropriate time intervals of lengths not less than a minimum residence time. A level switching law (supervisor) calculates the switching times subject to a residence time between the various estimators which is used as a mechanism to reparameterize on-line the basic adaptive controller in operation to generate the control input. The operation mode of such a supervisor is discussed in the sequel.

\subsection{Switching rule in the parallel multiestimation scheme (first-level supervision).} The objective of the supervisor mechanism is to evaluate the performance of the possible controllers connected to the plant with the aim of choosing the current controller from the set of parallel controllers. The subsequent supervision scheme selects on-line the consecutive switching times between the various estimators in order to reparameterize on-line the basic adaptive controller via the performance index

$$
J_{k}^{(\ell)}=\sum_{i=k-M}^{k} \lambda^{k-i}\left[\alpha\left(y_{i}-\hat{y}_{i}^{(\ell)}\right)^{2}+(1-\alpha)\left(\operatorname{Sup}_{0<j \leq i}\left(\sigma^{(\ell)^{i-j}}\left\|\varphi_{j}^{(\ell)}\right\|\right)\right)\right]
$$

for all $\ell \in N_{e}$, where $\hat{y}_{k}^{(\ell)}$ is the $\ell$ th predicted input given by

$$
\hat{y}_{k}^{(\ell)}=\left(1-\hat{A}_{k}^{(\ell)}\left(q^{-1}\right)\right) y_{k}+\hat{B}_{k}^{(\ell)}\left(q^{-1}\right) u_{k},
$$


where $0<\alpha \leq 1, \sigma^{(\ell)}$ was defined in Assumptions 2.2 (2.9), and $M$ is an integer number large enough to give sense to the performance evaluation. Note that (2.17) has two additive terms. The first one is a measure of the long-term accuracy of each identification algorithm, where the forgetting factor $\lambda \in(0,1]$ establishes the effective memory of the index in rapidly changing environments. The second one is introduced in order to make the system choose a reduced-order model with its unmodeled part being stable since $\sigma^{(\cdot)}<1$ in that case, while in a reduced-order model with its unmodeled part being unstable $\sigma^{(\cdot)}$ should be bigger than the unity. When $k$ is sufficiently large the second term will diverge for the model with unstable dynamics in its unmodeled part and then the system will choose only between the other ones. Now, the switching rule for the basic adaptive controller reparameterization is obtained from the performance index (2.17) as follows. Let the switching sampling times sequence, which may be finite or infinite countable, be denoted by $T S=\left\{t^{(1)}, t^{(2)}, \ldots, t^{(\pi)}\right\}$, where $\pi$ is the number of switchings with $\left(t^{(i+1)}-t^{(i)}\right) \geq \tau_{r}=N_{r} T$ (a known minimum residence time) for all $t^{(i)}, t^{(i+1)} \in T S$. Thus, the $c_{k}$-estimation scheme with $c_{k} \in N_{e}$, which parameterizes for all $k \geq 0$ the basic adaptive controller at any switching time in TS, is updated as follows. Assume that the last switching time for the controller reparameterization was $t^{(\pi)}$. Thus, for each current $k$-sampling time, define the auxiliary integer variable as in Algorithm 2.1.

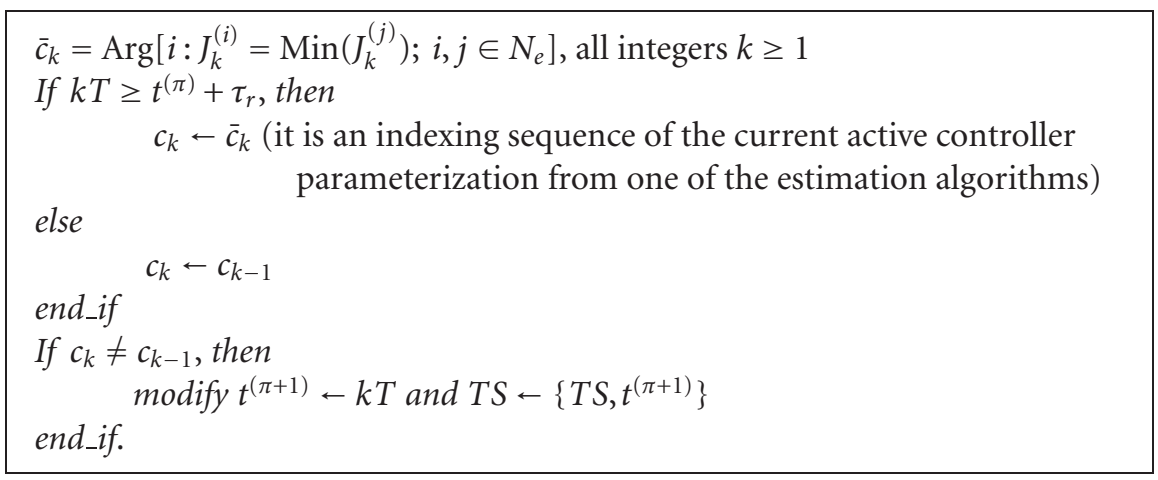

Algorithm 2.1

A minimum residence time that ensures the achievement of closed-loop stability always exists for any time-varying linear system consisting of a set of stable linear timevarying configurations $[1,3,4,8,11,19,20]$. Switches between those configurations at intervals exceeding such a time ensure the global stability. These ideas will be discussed in the next section related to the closed-loop stability supplied by the proposed adaptive controller based on a multiestimation scheme if the residence time is known. If it is unknown, since it always exists under weak assumptions, then it is estimated by successive on-line increments via closed-loop performance tests until an available upper bound is obtained in finite time so that stability is still ensured.

2.6. High-level supervision (second-level supervisor). A higher-level supervisor to the above one supervising the switching of parameterization for the active controller of potential optional use is now proposed. This new supervisor selects on-line the value of 
the $\alpha$ weighting factor, which is now time-varying as a result. The high-level supervision index is the following:

$$
J_{k}^{\prime}=\sum_{i=k-M^{\prime}}^{k}\left[\gamma\left(y_{i}-y_{i}^{m}\right)^{2}+(1-\gamma) u_{i}^{2}\right]
$$

where $y_{k}^{m}$ is the uniformly bounded reference output sequence and $u_{k}$ is the control signal (see Figure 2.4) for some prescribed weight $\gamma \in(0,1]$ such that if $k=j M^{\prime}$ for any nonnegative integer $j$, then $\alpha_{k+i}=\alpha_{k} \in\left[\alpha_{\min }, \alpha_{\max }\right] \subset(0,1], i=1,2, \ldots, M^{\prime}-1$, according to the following simple empirical rules: if $\alpha_{k}$ is increasing (decreasing) and the value of the performance index $J_{k}^{\prime}$ is decreasing, then continue with the same action on $\alpha_{k}$, otherwise change the updating action to decrease (increase) $\alpha_{k}$.

The conceptual idea is to continue with the same current correcting action if the tracking performance is improving and to change it otherwise. The current value of $\alpha_{k}$ is obtained by adding or subtracting a quantity $\Delta \alpha_{k}=\Delta \alpha_{k-M^{\prime}} / m f$ to $\alpha_{k-M^{\prime}}$ if $k=j M^{\prime}$. The integer $j$ should be bigger than unity since we need to wait two periods of $M^{\prime}$ to make the first comparison. The value $m f$ is a scaling factor, or modulation factor, which allows to increase/reduce the variation rate and which can optionally be reupdated on-line. Projection is used when the updated value lies outside $\left[\alpha_{\min }, \alpha_{\max }\right] \subset(0,1]$. The objective of the choice $M^{\prime} \neq M$ is to avoid both supervisions operating at the same speed which could lead to conflictive decisions. The experience from worked examples dictates that one of the horizons should be chosen of order double the size of the other. More formally, Algorithm 2.2 can be stated as follows.

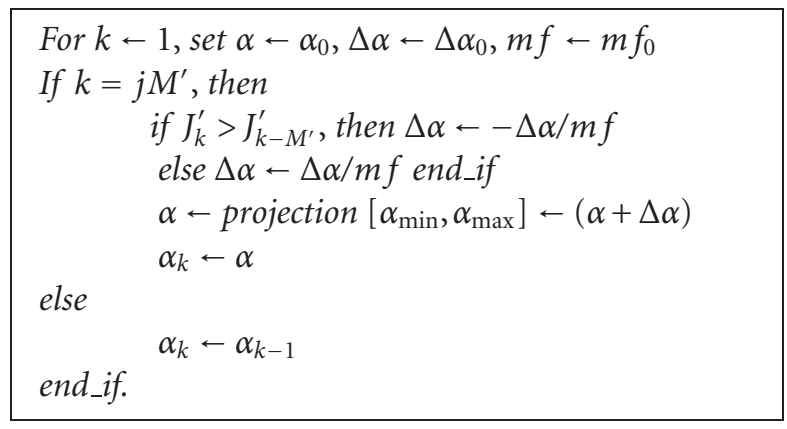

Algorithm 2.2

Note that if $M^{\prime} \geq M$, then the higher-level supervisor ( $\alpha$-supervision) operates at the same or a slower rate than the first-level supervisor (switching between estimator and controller parameterization pairs to decide the current active adaptive controller). Otherwise, the $\alpha$-supervision operates at a faster rate. The adjustment between both rates depends highly on the application and designer's a priori knowledge. Note that if in the initialization scheme of the above algorithm $\alpha_{\max }=\alpha_{\min }$, then the multiestimation scheme corresponds to the particular case of an (high-level) unsupervised multiestimation scheme with constant values. From Assumption 2.4, each polynomial diophantine 
equation of the form (2.16) for all $\ell \in N_{e}$ has a unique solution for all $k \geq 0$ under the degree constraints (2.15). The control law is

$$
R_{k} u_{k}=T u_{c k}-S_{k} y_{k}
$$

where $\left(R_{k}, S_{k}, T\right)=\left(R_{k}^{\left(c_{k}\right)}, S_{k}^{\left(c_{k}\right)}, T^{\left(c_{k}\right)}\right) \in\left\{\left(R_{k}^{(\ell)}, S_{k}^{(\ell)}, T^{(\ell)}\right) ; 1 \leq \ell \leq n_{e}\right\}$, that is, at each time, the tupla $\left(R_{k}, S_{k}, T\right)$ is defined at each sampling time by $\left(R_{k}^{(\ell)}, S_{k}^{(\ell)}, T^{(\ell)}\right)$ for some $\ell=$ $1,2, \ldots, n_{e}$ and then the control input is generated by the corresponding $\ell$ th adaptive controller parameterization as $u_{k}=u_{k}^{\left(c_{k}\right)}$ with $c_{k} \in N_{e}$, for all $k \geq 0$.

\section{Properties of the estimation and closed-loop stability}

3.1. Boundedness and convergence results of the parallel multiestimation scheme. In this work, all the recursive identification algorithms associated with the multiestimation scheme will be of standard least-squares type. For the multiestimation scheme, the following result proved in Appendix A follows.

THEOREM 3.1. The combined estimated parameter vector from the multiestimation scheme $\hat{\theta}_{k}=\hat{\theta}_{k}^{\left(c_{k}\right)}$ leading to the output estimate $\hat{y}_{k}=\hat{y}_{k}^{\left(c_{k}\right)}$ has the following properties for each $\ell \in$ $N_{e}$ irrespective of the control law:

(1) $\lim _{k \rightarrow \infty} s_{k}^{(\ell)}\left(e_{k}^{(\ell)^{2}}-\eta_{k}^{(\ell)^{2}}\right) /\left(1+\varphi_{k}^{(\ell)^{T}} P_{k}^{(\ell)} \varphi_{k}^{(\ell)}\right)\left(1+\left(1-s_{k}^{(\ell)}\right) \varphi_{k}^{(\ell)^{T}} P_{k}^{(\ell)} \varphi_{k}^{(\ell)}\right)=0$;

(2) $\lim _{k \rightarrow \infty} s_{k}^{(\ell)} \varphi_{k}^{(\ell)^{T}} P_{k}^{(\ell)} \varphi_{k}^{(\ell)}\left(e_{k}^{(\ell)^{2}}\left(1-s_{k}^{(\ell)}\right)-\eta_{k}^{(\ell)^{2}}\right) /\left(1+\varphi_{k}^{(\ell) T} P_{k}^{(\ell)} \varphi_{k}^{(\ell)}\right)(1+(1-$ $\left.\left.s_{k}^{(\ell)}\right) \varphi_{k}^{(\ell)^{T}} P_{k}^{(\ell)} \varphi_{k}^{(\ell)}\right)=0$

(3) $\sum_{k=0}^{\infty} s_{k}^{(\ell)} e_{k}^{(\ell)^{2}} /\left(1+\varphi_{k}^{(\ell)^{T}} P_{k}^{(\ell)} \varphi_{k}^{(\ell)}\right)<\infty ; \lim _{k \rightarrow \infty} s_{k}^{(\ell)} e_{k}^{(\ell)^{2}} /\left(1+\varphi_{k}^{(\ell)^{T}} P_{k}^{(\ell)} \varphi_{k}^{(\ell)}\right)=0$;

(4) If $\phi_{k}^{(\ell)}=s_{k}^{(\ell)} /\left(1+\varphi_{k}^{(\ell)^{T}} P_{k}^{(\ell)} \varphi_{k}^{(\ell)}\right)$, then $\phi_{k}^{(\ell)} e_{k}^{(\ell)^{2}} \rightarrow 0$ and $\phi_{k}^{(\ell)} \rightarrow 0$ as $k \rightarrow \infty$. If, in addition, $\left\|\varphi_{k}^{(\ell)}\right\|$ is bounded for all $k \geq 0$, then $s_{k}^{(\ell)} e_{k}^{(\ell)^{2}} \rightarrow 0$ and $s_{k}^{(\ell)} \rightarrow 0$ as $k \rightarrow \infty$.

(5) $\left\|\tilde{\theta}_{k}^{(\ell)}\right\|<\infty ;\left\|\hat{\theta}_{k}^{(\ell)}\right\|<\infty$. Moreover, $\hat{\theta}_{k}^{(\ell)} \rightarrow \hat{\theta}_{\infty}^{(\ell)}$ (finite) and $\tilde{\theta}_{k}^{(\ell)} \rightarrow \tilde{\theta}_{\infty}^{(\ell)}$ (finite) as $k \rightarrow \infty$.

Note that every controller parameter vector is bounded for all $k \geq 0$ and has a finite limit as $k \rightarrow \infty$ for all $\ell \in N_{e}$ from the boundedness of all the estimates of the parallel multiestimation scheme. Note that Theorem 3.1 holds irrespective of the control law and on the switching rate between the various adaptation algorithms within the parallel multiestimation scheme.

3.2. Closed-loop stability. Now, let $\hat{A}_{k}=\hat{A}_{k}^{\left(c_{k}\right)}, \hat{B}_{k}=\hat{B}_{k}^{\left(c_{k}\right)}, R_{k}=R_{k}^{\left(c_{k}\right)}, S_{k}=S_{k}^{\left(c_{k}\right)}$, and $T=$ $T^{\left(c_{k}\right)}$ the plant estimation and controller polynomials, $c_{k} \in N_{e}$ being the current estimator/controller pair in operation. In order to prove the closed-loop stability, the following auxiliary $(3 n-1)$ th linear time-varying extended system is used for subsequent analysis:

$$
x_{k+1}=G_{k} x_{k}+\vartheta\left(e_{k}+v_{k}\right)
$$

where $v_{k}=T\left(q^{-1}\right) u_{c k}$ is a uniformly bounded forcing signal and

$$
x_{k}^{T}=\left[\begin{array}{lll}
y_{k} & y_{k-1} \cdots y_{k-n+1} & u_{k} \cdots u_{k-2 n+1}
\end{array}\right]
$$


is the state vector, while $G_{k}=G_{k}^{\left(c_{k}\right)}$, which is built with $\hat{A}_{k}=\hat{A}_{k}^{\left(c_{k}\right)}, \hat{B}_{k}=\hat{B}_{k}^{\left(c_{k}\right)}, R_{k}=R_{k}^{\left(c_{k}\right)}$, and $S_{k}=S_{k}^{\left(c_{k}\right)}$ as follows:

$$
\begin{aligned}
& G_{k}=G_{k}^{\left(c_{k}\right)} \\
& =\left[\begin{array}{ccccccc|cccccc}
-\hat{a}_{1 k} & -\hat{a}_{2 k} & & \cdots & -\hat{a}_{i, k} 0 & \cdots & 0 & \hat{b}_{0 k} & \hat{b}_{1 k} & \cdots & \hat{b}_{i-1, k} 0 & \cdots & 0 \\
1 & 0 & & & & & 0 & 0 & 0 & & & & 0 \\
0 & 1 & & & & & 0 & \vdots & \vdots & \ddots & & & \vdots \\
\vdots & \vdots & & \ddots & & & \vdots & & & & & & \\
0 & 0 & & \cdots & 1 & & 0 & 0 & 0 & & \cdots & & 0 \\
\hline-s_{0 k} & -s_{1 k} & -s_{2 k} & \cdots & -s_{i-1, k} 0 & \cdots & 0 & -r_{1 k} & -r_{2 k} & \cdots & -r_{i k} 0 & \cdots & 0 \\
0 & 0 & 0 & \cdots & 0 & & 0 & 1 & 0 & \cdots & 0 & & 0 \\
\vdots & \vdots & \vdots & \ddots & \vdots & & \vdots & \vdots & \vdots & \ddots & \vdots & & \vdots \\
0 & 0 & 0 & \cdots & 0 & \cdots & 0 & 0 & 0 & \cdots & 1 & \cdots & 0
\end{array}\right]
\end{aligned}
$$

for the current active $\ell=c_{k} \in N_{e}$ at the $k$ th sampling instant, is the matrix of the dynamics associated with the current estimator-controller pair in operation, and $9^{T}=$ $\left[\begin{array}{llll}1 & 0 & \cdots & 0\end{array}\right]$. The subsequent technical assumption and result are given to be later used to prove the scheme's closed-loop stability.

Assumption 3.2. Assume that for any integers $k_{0} \geq 0$ and $k \geq k_{0}+1, \sum_{j=k_{0}+1}^{k} \| G_{j}^{(\ell)}-$ $G_{j-1}^{(\ell)} \|^{2} \leq \beta_{0}^{(\ell)}+\beta_{1}^{(\ell)}\left(k-k_{0}\right)$ for some positive real constants $\beta_{0}^{(\ell)}$ and $\beta_{1}^{(\ell)}$ and all $\ell \in N_{e}$.

Lemma 3.3. (i) If Assumption 3.2 holds, then $\prod_{j=k_{0}+1}^{k}\left\|G_{j}^{(\ell)}\right\| \leq K^{(\ell)} \rho_{0}^{k-k_{0}}$, where $K^{(\ell)} \geq 1$ and $\rho_{0}$ is the convergence abscissa of all $G_{k}^{(\ell)}$ provided that $\beta_{1}^{(\ell)}$ are sufficiently small for all $\ell \in N_{e}$.

(ii) If, in addition, the residence time $\tau_{r}=N_{r} T$ is sufficiently large, then $\prod_{j=k_{0}+1}^{k}\left\|G_{j}^{\left(c_{k}\right)}\right\| \leq$ $K \rho^{k-k_{0}}$ for some $K \geq 1$ and $\rho \in\left(\rho_{0}, 1\right)$, where $N_{e}^{\prime}$ is a subset of $N_{e}$ with one of its elements deleted.

Proof of Lemma 3.3. For the proof of (i), see [12]. To prove (ii), define the set of indexes $I T S\left(k_{0}+1, k\right)=\operatorname{Arg}\left(k \in \mathbb{Z}: t_{k} \in\left[\left(k_{0}+1\right) T, k T\right] \cap T S\right)$ that characterize the sampling instants where switchings between estimation schemes take place within the time interval $\left[\left(k_{0}+1\right) T, k T\right]$. Now, for the given $\rho_{0}$, choose $\rho>\rho_{0}$ such that $\left(\rho_{0} / \rho\right)^{N_{r}} K^{(\ell)} \leq 1$ for all $\ell \in N_{e}^{\prime}$. Note that such a choice is always possible for sufficiently large residence time $\tau_{r}$. Thus, the result follows from (i) by choosing $K=K^{(\ell)}$ for the unique estimator/controller parameterization pair $\ell \in\left(N_{e}-N_{e}^{\prime}\right)$ since

$$
\prod_{j=k_{0}+1}^{k}\left[\prod_{\ell \in I T S\left(k_{0}+1, k\right)} K^{(\ell)}\left(\frac{\rho_{0}}{\rho}\right)^{k-k_{0}}\right] \rho^{k-k_{0}} \leq K \rho^{k-k_{0}} .
$$

The proof has been completed.

Note in the proof of Lemma 3.3 that $\rho>\rho_{0}$ is chosen so that $\left(\rho_{0} / \rho\right)^{\tau_{r} / T} K^{(\ell)} \leq 1$ for all $\ell \in N_{e}^{\prime}$, then Lemma 3.3(ii) holds for $K=1$. From the structure of the matrices (3.2b) for 
$\ell \in N_{e}$, Assumption 3.2 means that the estimates parameter vector varies asymptotically slowly from one sample to another, a condition which is satisfied trivially by the proposed estimation algorithm because the estimated vector converges to a finite limit. This fact leads to the subsequent robust main closed-loop stability result which is proved in Appendix B.

Theorem 3.4 (closed-loop stability in the case when at least one of the reduced-order models possesses associated stable unmodeled dynamics). The extended system (3.1)(3.2) and the closed-loop adaptive system involving the parallel multiestimation scheme (2.12) with the pole-placement-based adaptive control law (2.20) are both globally stable provided that Assumptions 2.1, 2.2, 2.4, and 3.2 hold with the constant $\alpha_{1}^{\prime}=\operatorname{Max}_{\ell \in N_{e s}}\left(\alpha_{1}^{(\ell)}+\right.$ $\left.\left\|\tilde{\theta}_{\infty}^{(\ell)}\right\|\right) \geq 0$ being sufficiently small satisfying $\alpha_{1}^{\prime} \leq(1-\rho-\vartheta) / K$ for any real prefixed constant $\vartheta \in(0,1-\rho)$ and that the residence time for each current estimation scheme in operation is sufficiently large satisfying $N_{r} \geq\left(\ln K+\vartheta^{\prime}\right) /\left(\ln \rho-\ln \rho_{0}\right)$ for any prefixed real constant $\vartheta^{\prime}>0$. If the transfer function describing the unmodeled dynamics is strictly proper then the above constraints may be relaxed to $\alpha_{1}^{(\ell)} \leq(1-\rho-\vartheta) / k \sigma$ for all $\ell \in N_{e}$.

A particular case of Theorem 3.4, which does not require a specific proof given in detail in [8], is concerned with the case when all the reduced-order models have associated stable unmodeled dynamics and it is established below.

Corollary 3.5 (closed-loop stability in the case when all the reduced-order models possess associated stable unmodeled dynamics). Theorem 3.4 holds for the case when $N_{e}=N_{e s}$ and $N_{e u}=\varnothing$ (i.e., all the estimators have associated stable unmodeled dynamics).

Note that the above result ensures robust closed-loop stability for certain small amounts of unmodeled dynamics in the plant. Note that in the current approach, the estimators are related to reduced-order models while in the previous ones in the literature the estimators were arbitrary nonnecessary linked to particular reduced-order models. Note also that it is required that only one of the reduced-order models have stable unmodeled dynamics (Theorem 3.4) contrary to previous assumptions in the light of Corollary 3.5, that is, by assuming that all the reduced-order models possess stable unmodeled dynamics. The unmodeled dynamics must grow nonlinearly faster than linearly with their associated regressors at sufficiently small rates (i.e., the maximum of the $\alpha_{1}^{(\ell)}$ is small) provided that the time interval between two consecutive estimation switchings is sufficiently large $\left(\geq \tau_{r}\right)$ if Assumption 3.2 holds (i.e., the increments of the estimated parameters grow nonlinearly faster than linearly with time at sufficiently small rate). Note that the precise knowledge of the minimum residence time $\tau_{r}$ is not necessary since it may be increased on-line from an initial estimation until an upper bound is reached compatible with the closed-loop stability. This property may be tested on-line for particular worked examples.

\section{Simulation results}

4.1. Simulation 1 (multiestimation scheme without high-level supervision and unstable unmodeled dynamics). In this section, some exhaustive numerical experimentation has been worked for the unstable controller plant of transfer function 


$$
H(z)=\frac{2 z^{4}-2.125 z^{3}+0.1687 z^{2}+0.2575 z-0.08169}{z^{5}-1.85 z^{4}+0.73 z^{3}-0.005 z^{2}+0.07 z-0.04063}
$$

Using the proposed decomposition in (2.5), the plant transfer function may be rewritten as

$$
H(z)=\frac{0.25}{z-0.5}+\frac{0.125}{(z-0.5)^{2}}+\frac{0.75 z+0.375}{z^{2}+0.4 z+0.13}+\frac{0.5}{z-1.25}
$$

For the multiestimation scheme three reduced-order models of the plant are proposed. The first one considers the pole of multiplicity two in 0.5 and the pair of complex conjugate poles. The second reduced-order model does not consider the complex conjugate pair and the third one removes the two real poles in 0.5 . The estimated reduced-order plant models could be represented as follows:

$$
\begin{aligned}
& \hat{H}^{(1)}(z)=\frac{\hat{k}_{1,1}^{(1)}}{z-0.5}+\frac{\hat{k}_{1,2}^{(1)}}{(z-0.5)^{2}}+\frac{\hat{f}_{2,1}^{(1)} z+\hat{g}_{2,1}^{(1)}}{z^{2}+0.4 z+0.13}, \\
& \hat{H}^{(2)}(z)=\frac{\hat{k}_{1,1}^{(2)}}{z-0.5}+\frac{\hat{k}_{1,2}^{(2)}}{(z-0.5)^{2}}+\frac{\hat{k}_{3,1}^{(2)}}{z-1.25}, \\
& \hat{H}^{(3)}(z)=\frac{\hat{f}_{2,1}^{(3)} z+\hat{g}_{2,1}^{(3)}}{z^{2}+0.4 z+0.13}+\frac{\hat{k}_{3,1}^{(3)}}{z-1.25}
\end{aligned}
$$

Note that in the first reduced-order model the unmodeled part is unstable. The stable reference model transfer function is $H_{m}(z)=0.3375\left(z^{2}-0.5 z\right) /\left(z^{3}-1.1 z^{2}+0.2875 z-\right.$ $0.01875)$. The sampling period is fixed to $T_{s}=0.1$ second. A parallel multiestimation scheme with three estimation/adaptive controller parameterization pairs has been used with the precompensator and feedback compensator including an integrator. The performance tests of the tracking and control efficiency of the adaptive scheme have been performed by using high-level supervision (i.e., on-line updating of the $\alpha$-weights) or not (prefixed $\alpha$-weight while only using switchings between estimators) and different reference inputs as unity steps, square waves, and sinusoids. The used residence time is an upper bound of the real one and it is found by numerical testing by varying it gradually as the estimation algorithm evolves with time with small increments from smaller to larger tentative values. In this context, no extra computation is needed but one only uses the knowledge that a minimum residence time exists (see Lemma 3.3 and Theorem 3.4). The adaptation relative dead-zone algorithm is applied with $\mu=\mu^{(\ell)}=$ 1.005 for $\ell=1,2,3$ and the other parameters are chosen as $\sigma^{(1)}=1.3, \sigma^{(2)}=\sigma^{(3)}=0.95$, $\alpha_{1}^{(1)}=2, \alpha_{1}^{(2)}=0.025, \alpha_{1}^{(3)}=0.075, \alpha_{0}^{(1)}=2, \alpha_{0}^{(2)}=0.025$, and $\alpha_{0}^{(3)}=0.075$. The initial parameters of the performance indexes are chosen as $\lambda=\alpha=0.95$. The residence time is 10 
samples. The initial conditions for the three estimation algorithms are $\hat{\theta}_{0}^{(1)}=(1,1,1,1)^{T}$, $\hat{\theta}_{0}^{(2)}=(1,1,1)^{T}, \hat{\theta}_{0}^{(3)}=(1,1,1)^{T}$ and the three $P_{0}^{(\ell)}$ are $\operatorname{Diag}\left(10^{15}\right)$ of appropriate orders. In the first simulation a unity step signal is used as reference input. Figure 4 .1a shows the reference and plant outputs. Figure $4.1 \mathrm{~b}$ shows the active controller at each time interval. The scheme is arbitrarily initialized in the third controller, but it chooses the one with the best performance. In this case, the second one gives the best response. We can see as well how there is a difference between the desired output and the real one due to the fact that the estimation is stopped when the identification error is sufficiently small.

In Figures 4.1c and 4.1d, we can observe the unmodeled part contributions and the upper bound used in the dead zone for the second and third controllers. Note that the true value is always smaller.

Figure 4.1e shows the estimated output for each estimator. Note that the second estimator gives the closer estimated output to the real one. Finally, Figure 4.1f shows the estimated parameters for the selected controller, which turns out to be the second one.

In the next simulation, the reference input is changed. Now a sinusoidal signal with $T_{\text {ref }}($ period $)=200$ samples is used. Figure $4.2 \mathrm{a}$ shows the plant output and the reference one. We can see in Figure 4.2b that in this case the scheme chooses the third controller parameterization after a small number of switches between estimators/adaptive controller pairs. Figures $4.2 \mathrm{c}$ and $4.2 \mathrm{~d}$ show the contribution of the unmodeled parts and the upper bounds used in the designed dead zone for the second and third estimators. Finally, Figure 4.2e shows the estimated output for each estimator with the real plant output.

Figure 4.2e shows how the estimated output of the second estimator is closer than the estimated output of the third one. However, the scheme chooses the last one. This happens because the performance index depends not only on the identification error but also on the additional term which has been added in order to make the system choose a reduced-order model with a stable unmodeled part. The importance of this term is given by the value of the weighting factor $\alpha_{k}$. In this case, we can improve the scheme response giving more importance to the identification error in the supervisory index. This is made by increasing the value of $\alpha_{k}$. In the following simulation, this parameter is fixed to 0.995. Simulation (Figures $4.2 \mathrm{f}$ and $4.2 \mathrm{~g}$ ) now shows how the scheme chooses the second estimator/controller pair which had the best identification performance in the previous simulation. In this case, the obtained output is much better than in the previous case. The last simulation shows the importance of finding an appropriate value for the weighting factor $\alpha_{k}$. We can introduce a supervision which updates on-line its value in order to find the best one.

\subsection{Simulation 2 (multiestimation scheme without high-level supervision and stable} unmodeled dynamics). In this simulation the following stable plant is used:

$$
\begin{aligned}
H(z) & =\frac{0.25}{z-0.5}+\frac{0.125}{(z-0.5)^{2}}+\frac{0.75 z+0.375}{z^{2}+0.4 z+0.13}+\frac{0.5}{z+0.8} \\
& =\frac{1.5 z^{4}+0.225 z^{3}-0.385 z^{2}-0.4525 z+0.09125}{z^{5}+0.2 z^{4}-0.5 z^{3}-0.046 z^{2}+0.0085 z+0.026}
\end{aligned}
$$




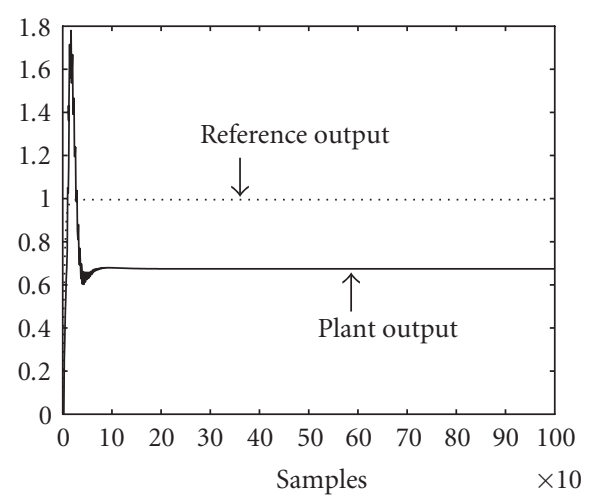

(a)

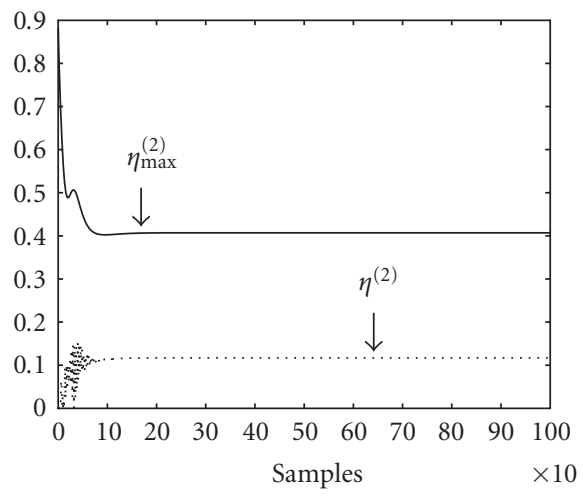

(c)

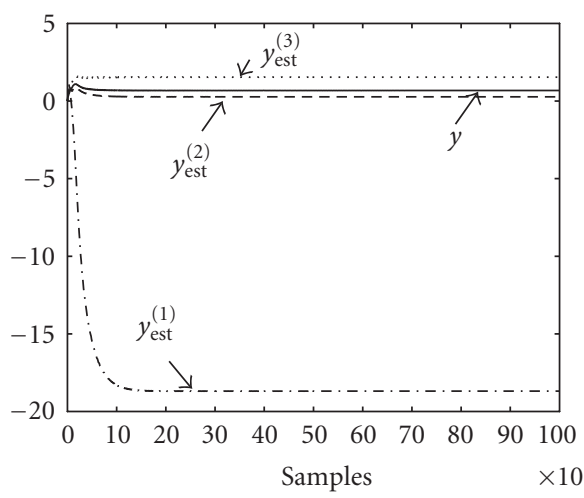

(e)

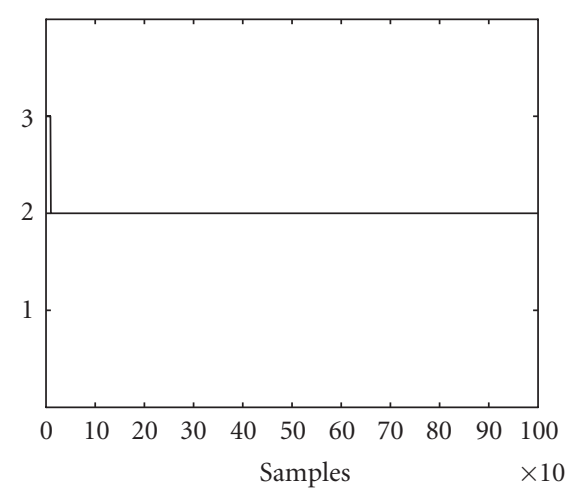

(b)

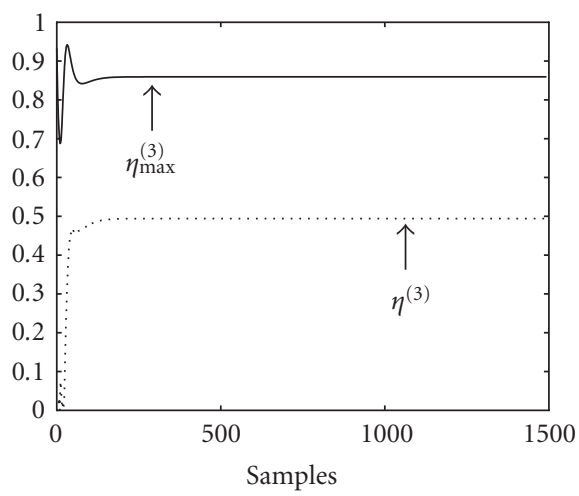

(d)

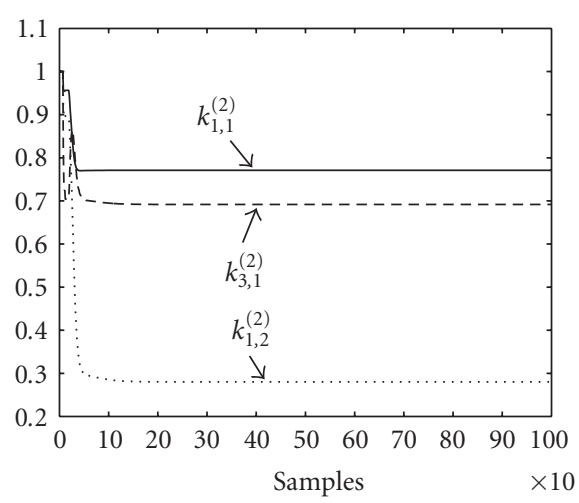

(f)

Figure 4.1. (a) Reference and plant outputs when the high-level unsupervised multiestimation scheme is used for a unity step input signal. (b) Active controller. (c) Contribution of the unmodeled part in the second model and the applied upper bound. (d) Contribution of the unmodeled part in the third model and the applied upper bound. (e) Estimated outputs and plant output. (f) Estimated parameters for the second estimator. 


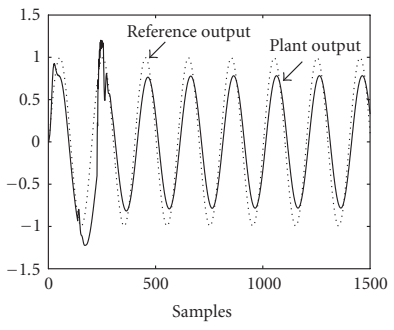

(a)

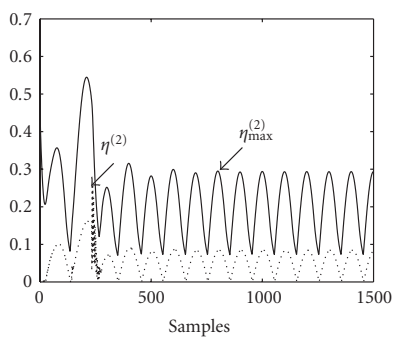

(c)

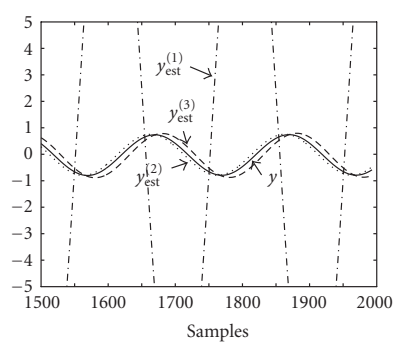

(e)

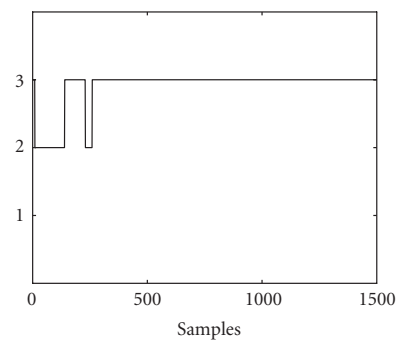

(b)

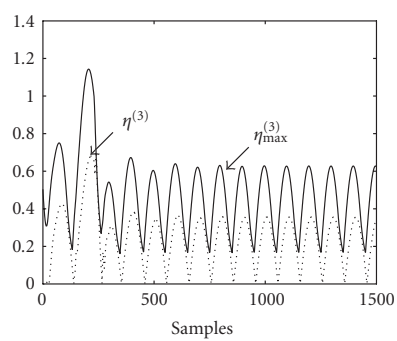

(d)

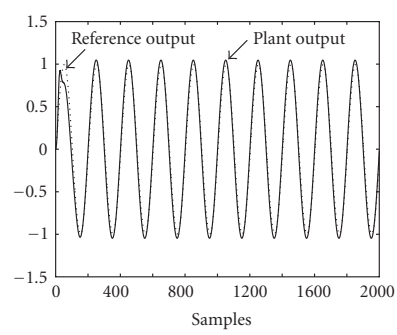

(f)

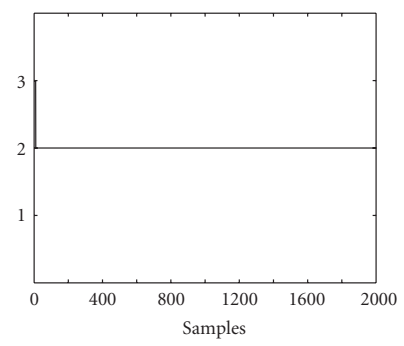

(g)

Figure 4.2. (a) Plant and reference outputs with $\alpha_{k}=0.95$. (b) Active controller. (c) Contribution of the unmodeled part in the second model and the applied upper bounds. (d) Contribution of the unmodeled part in the third model and the applied upper bounds. (e) Estimated outputs over the last 500 simulation samples. (f) Plant and reference outputs with $\alpha_{k}=0.995$. (g) Active controller. 
Since there is no unstable pole in the plant, all the proposed reduced-order models have associated stable unmodeled dynamics and instead of Theorem 3.4, Corollary 3.5 is applied. The reduced-order models could be written in the following way:

$$
\begin{aligned}
& \hat{H}^{(1)}(z)=\frac{\hat{k}_{1,1}^{(1)}}{z-0.5}+\frac{\hat{k}_{1,2}^{(1)}}{(z-0.5)^{2}}+\frac{\hat{f}_{2,1}^{(1)} z+\hat{g}_{2,1}^{(1)}}{z^{2}+0.4 z+0.13}, \\
& \hat{H}^{(2)}(z)=\frac{\hat{k}_{1,1}^{(2)}}{z-0.5}+\frac{\hat{k}_{1,2}^{(2)}}{(z-0.5)^{2}}+\frac{\hat{k}_{3,1}^{(2)}}{z+0.8}, \\
& \hat{H}^{(3)}(z)=\frac{\hat{f}_{2,1}^{(3)} z+\hat{g}_{2,1}^{(3)}}{z^{2}+0.4 z+0.13}+\frac{\hat{k}_{3,1}^{(3)}}{z+0.8} .
\end{aligned}
$$

For the dead zone, the estimation algorithm uses the following parameters: $\sigma^{(1)}=\sigma^{(2)}=$ $\sigma^{(3)}=\sigma=0.95, \alpha_{1}^{(1)}=0.1, \alpha_{1}^{(2)}=0.3, \alpha_{1}^{(3)}=2, \alpha_{0}^{(1)}=0.03, \alpha_{0}^{(2)}=0.06$, and $\alpha_{0}^{(3)}=0.3$, and it is initialized as $\hat{\theta}_{0}^{(1)}=(1,1,1,1)^{T} ; \hat{\theta}_{0}^{(2)}=(1,1,1)^{T} ; \hat{\theta}_{0}^{(3)}=(1,1,1)^{T}$ and the three $P_{0}^{(\ell)}$ are $\operatorname{Diag}\left(10^{15}\right)$ of appropriate orders. The initial parameters of the performance indexes are fixed to $\lambda=\alpha=0.95$. The residence time is 20 samples. The same reference transfer function as in the previous simulation is proposed. In this simulation, a sinusoidal signal with 200 samples period is used as reference input. Figure 4.3a shows the plant output and the reference one. The evolution of the active controller at each sampling time is shown as well in Figure 4.3b.

Figure $4.3 \mathrm{c}$ shows the evolution of the unmodeled dynamics for the first reduced-order model with the proposed upper bound for the design of the dead zone. The same figures for the second and third reduced-order models are omitted since they are similar to the ones shown.

We can observe the evolution of the estimates for each reduced-order model in Figures $4.3 \mathrm{~d}, 4.3 \mathrm{e}$, and $4.3 \mathrm{f}$. Figure $4.3 \mathrm{~g}$ shows the estimated outputs for the three different estimators. The real output is plotted as well in that figure.

4.3. Simulation 3 (multiestimation scheme with high-level supervision). In this simulation the high-level supervision is applied in order to find the most appropriate value for the $\alpha$-weighting factor. In the first simulation, we saw how the value of this parameter was relevant in the performance of the scheme. Two different values for this factor were used for the same simulation and we obtained an important difference between both cases, because with the inappropriate value the system did not choose the best reduced-order model. Now we will see how the supervision solves this problem. The same plant and reference inputs as in the first simulation are used, maintaining all the parameters with the same values. In the supervision of $\alpha$, it is initialized in 0.95 , while $\Delta \alpha_{0}=0.2, m f_{k}=1.1$ for all $k \geq 0$ and $\alpha_{k} \in[0.75,1]$. The value is updated every 20 samples. Figure $4.4 \mathrm{a}$ shows the plant output and the reference one. The evolution of the active controller is shown as well in Figure 4.4b. In this case, starting from an arbitrary value $\alpha_{0}$, we have achieved a good performance by updating this value every 20 samples (see Figure $4.4 \mathrm{c}$ ). Once the system has found an appropriate value for the weighting factor, the system can select the best reduced-order model resulting in better response compared to the case of the fixed value of $\alpha_{k}$ to 0.95 (Figure $4.2 \mathrm{a}$ ). 
A. Bilbao-Guillerna et al. 51

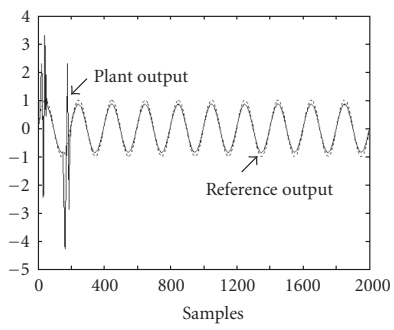

(a)

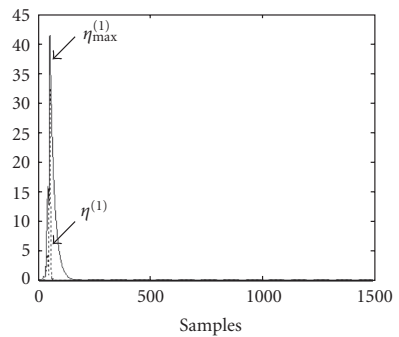

(c)

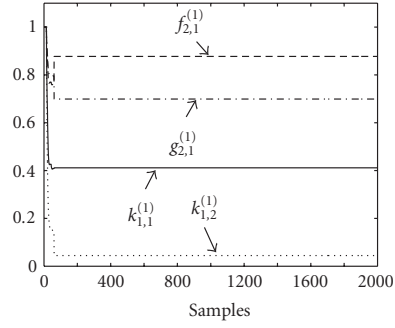

(d)

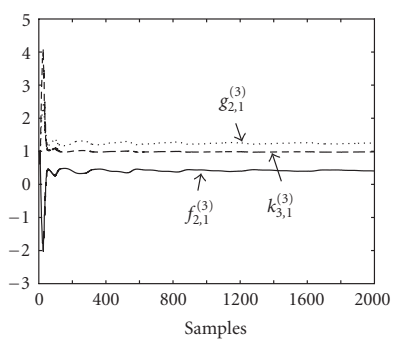

(f)

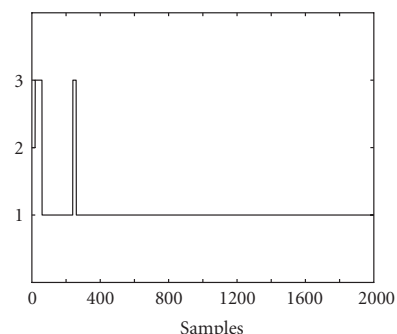

(b)
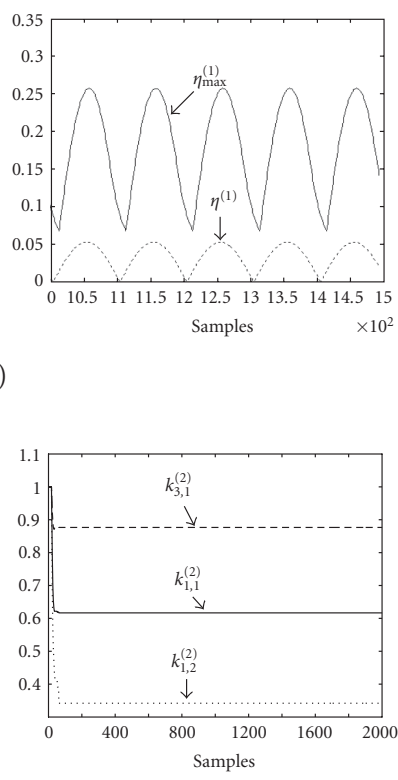

(e)

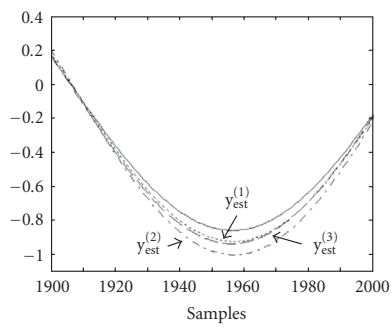

(g)

Figure 4.3. (a) Reference and plant outputs. (b) Active controller. (c) Contribution of the unmodeled part in the first model and the applied upper bounds. (d) Evolution of the estimates in model 1. (e) Evolution of the estimates in model 2. (f) Evolution of the estimates in model 3. (g) Estimated outputs for each estimator. 
52 Multiestimation for adaptive control and reduced models

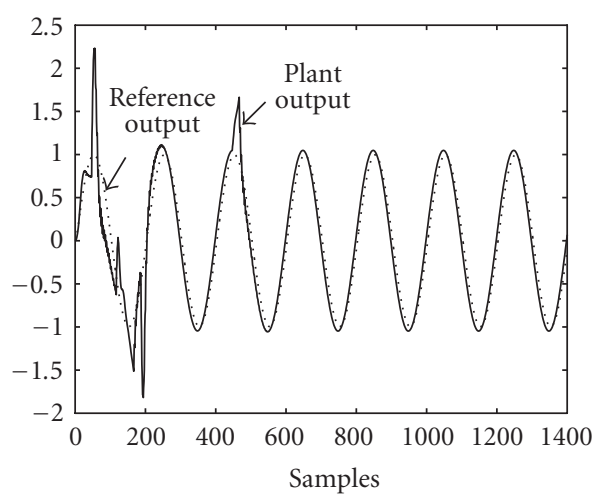

(a)

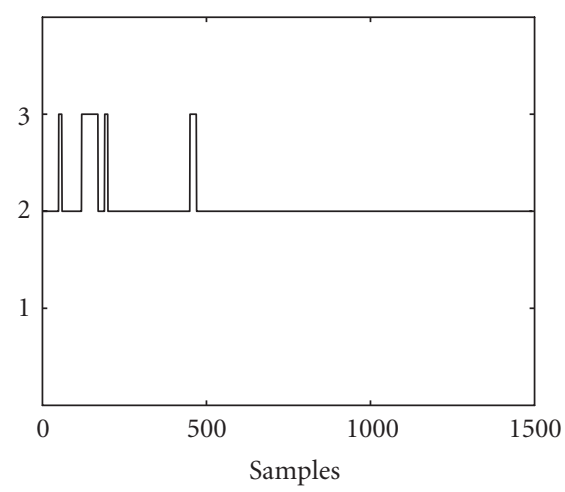

(b)

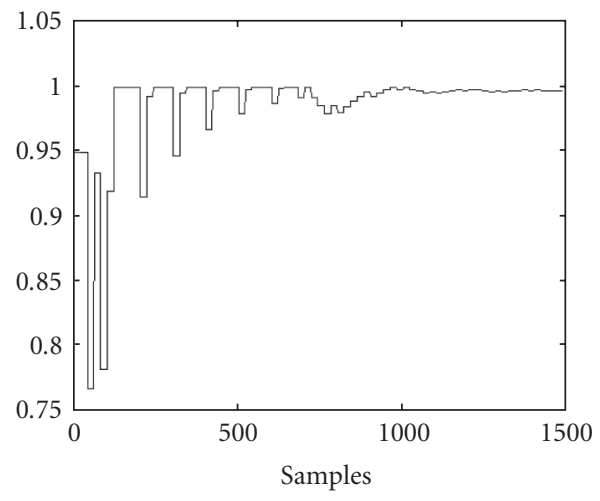

(c)

Figure 4.4. (a) Plant output and reference output. (b) Active controller. (c) Evolution of $\alpha$-weighting factor.

\section{Identification}

5.1. Introduction. In this section, a method for obtaining a plant-simplified model based on the input dominant frequencies is proposed. The idea is to integrate the on-line model order choice with the reference input spectrum by means of a switching rule between several plant recursive identifiers of different orders which run in parallel within a multiestimation scheme. The overall process is stated as an automatic task that does not require any on-line designer operation except the choice of the free-design parameters of the identifiers including their initializations. It is well known that initializations are crucial for the convergence rate in ARMA models or for the need to invert the transfer function at frequencies where it is very small in Wiener and Hammerstein systems [8]. The time intervals between consecutive switches are subject to a minimum residence time that guarantees an acceptable transient behavior $[1,3,4,6,8,16,20]$. The objective of this section is 
to identify online a reduced-order estimated model for a real plant under different operation points, which depend on the frequency of the input signal applied to the system. The procedure to achieve this objective consists of designing a multiestimation scheme composed of a set of estimators running in parallel. Such estimation algorithms run in parallel and each of them estimates the parameters of each proposed nominal plant model. Each of these nominal models represents one of the possible operation points of the real plant. They can be of different orders and all of them are underparameterized. In this way, a reduced-order estimated model for the current operation point of the plant could be obtained linked with the properties of one external input at each current time interval. The various different nominal models are built as parallel connections of distinct-order filters related to the set of modes of the plant, which are supposed to be known. However, the numerators of those filters are unknown so that they have to be recursively estimated. In summary, all of the considered nominal models contain some of the modes, but potentially not all, of the real plant and they are estimated separately within a multiestimation scheme. A supervisor incorporating a suitable switching law selects on-line the "best" estimation model that optimizes an appropriate cost function which depends on the error signal between the real output signal and the issued output from each estimated model. Since all the estimation algorithms included in the multiestimation scheme can provide an underparameterized estimation model of the plant, each plant identifier is potentially subject to the presence of unmodeled dynamics associated with the modes which are not included in its corresponding nominal model. Then, a suitable adaptation dead zone can be added in each estimation algorithm to improve the identification process. However, the estimation efficiency depends on the size of the unmodeled dynamics along time for each of the parallel identifiers, as will be illustrated by means of some simulation results.

\subsection{Theoretical setting up}

5.2.1. Main ideas and plant assumptions. The behavior of a discrete-time nonlinear plant around any operation point can be described by means of the time-invariant difference equation (2.6), subject to the parameterization (2.7). This is another specific interpretation of a practical situation where the multiestimation technique may be of usefulness in an identification context. Thus, note that (2.6) can describe the behavior around any operation point of the system resulting from the discretization via a sampling and hold device of a continuous-time plant. The following assumption, which is more appropriate in identification context, replaces Assumption 2.1.

Assumptions 5.1. (i) The plant modes are stable (since the system is assumed to operate in open loop) and known.

(ii) The nominal transfer function associated with each operation point does not necessarily include all the modes of the plant.

Assumptions 5.1(i) imply that the roots of the polynomials $A^{(\ell)}$, and then its coefficients, associated with each operation point are known. However, the parameters of the polynomials $B^{(\ell)}$ will be not perfectly known or even unknown and then an estimation algorithm will have to be used for identification purposes. Assumptions 5.1(ii) are introduced in order to obtain a reduced-order nominal model of the plant depending 
on the environment which justifies the use of different order models depending on the current operating point. Such an assumption is not necessary to set the mathematical properties of the estimation while it is convenient in an application setting up context. Therefore, the behavior of the plant at each operation point can be nominally modeled as a parallel connection of strictly proper filters, with poles belonging to a subset of the modes of the plant, of unknown numerator factors.

5.2.2. Plant description at different operation points. In view of Assumptions 5.1 the nominal model associated with the behavior of the plant at the $\ell$ th operation point can be described by means of the transfer function (2.7) (see Figure 2.1). The main idea consists of eliminating some of the modes of the plant to obtain a reduced-order model of the plant in the current operation point. The main motivation is that a full description of all modes of the plant might not be necessary for describing the behavior of the plant for each current input during some time intervals. For instance, the excitation of some of the plant modes can be very small with respect to other ones for certain frequency ranges. Then, such modes were not considered in (2.7) for describing the plant behavior subject to inputs of frequency spectrum included in the aforementioned range. In this way, a reduced-order nominal model of the plant could be obtained including the relevant modes. In the whole estimation scheme, a number $n_{e}$ of plant nominal models will be considered, one for each possible plant operating condition. Each of them does not contain at least one of the plant modes. Such unincluded modes constitute the unmodeled dynamics associated with the corresponding nominal model. The subsequent assumption is considered about the unmodeled dynamics.

Assumption 5.2. The contribution to the plant output of the unmodeled dynamics associated with each nominal model can be expressed as a sum of a bounded term plus a term related to the input by a strictly proper exponentially transfer function.

The transfer function mentioned in Assumption 5.2 is the result of the sum of different order filters related with the plant modes which are not included in each plant nominal model, that is, the modes not included in each formula of the form (2.7). Such filters are stable in view of Assumption 3.2(i). Therefore, Assumption 5.2 is met by the description of the plant behavior at any operation point given by (2.6). Such an assumption ensures the existence of an upper bound for the contribution of the unmodeled dynamics to the plant output $[1,8,15]$.

\subsection{Multiestimation scheme}

5.3.1. Motivation and description of the identification process. A multiestimation technique is used to obtain an estimation model of the plant including a set of different nominal models, each one associated with each possible plant operation point. Each estimator works separately and in parallel while estimating the parameters of its associated nominal model. The main idea is to know which of the estimated models is the best approach to the behavior of the plant at each instant according to identification quality dictated by an identification error performance index. Obviously, the model which better represents the plant behavior normally changes through time since it depends on the operation condition of the plant. It becomes apparent that there exists a need to make a comparison 
among the identification performances associated with all estimated models issued by the multiestimation scheme at certain time instants to know which is the most approximated to the plant in the current instant. For such a purpose, the identification performance index (2.16), which compares the true plant output $y_{k}$ with the estimated one by the corresponding estimated model $\hat{H}^{(\ell)}(z)$, is particularized with the weight $\alpha$ fixed to unity. Obviously, the estimated model with the smallest value for the index $J_{k}^{(\ell)}$ is the best approximation to the true plant behavior for the current time interval. Note that the estimation can be performed because the real output does not diverge since the true plant is stable.

5.3.2. Estimation algorithms. Two different estimation algorithms are proposed to identify the unknown parameters $f_{j, i}, g_{j, i}$, and $k_{j, i}$ of the nominal models which conform the multiestimation scheme. One of the algorithms does not incorporate any tools to treat with the unmodeled dynamics associated with each nominal model. However, the other one includes a relative adaptation dead zone in order to improve the identification process under the presence of unmodeled dynamics. Such a dead zone makes the adaptation process slower and stops it when the identification error is close to the uncertainty related to the unmodeled dynamics. The uncertainty from the unmodeled dynamics can be measured by a known upper-bounded function of its contribution to the output [9]. The motivation for considering two algorithm types is to compare the identification performance reached with both alternatives and to show how such a performance depends on the magnitude of the unmodeled dynamics.

Algorithm 5.3 (without dead zone). A standard least-squares algorithm is considered for adapting the parameters $\hat{k}_{j, i}^{(\ell)}, \hat{f}_{j, i}^{(\ell)}$, and $\hat{g}_{j, i}^{(\ell)}$ for $j \in\left\{1,2, \ldots, n_{r}^{\prime}+n_{c}^{\prime} / 2\right\}, i \in\left\{1,2, \ldots, \partial_{j}\right\}$, and $\ell \in N_{e}$ as follows:

$$
\begin{gathered}
\hat{\theta}_{k+1}^{(\ell)}=\hat{\theta}_{k}^{(\ell)}+\frac{P_{k}^{(\ell)} \varphi_{k}^{(\ell)} e_{k}^{(\ell)}}{1+\varphi_{k}^{(\ell)^{T}} P_{k}^{(\ell)} \varphi_{k}^{(\ell)}}, \\
P_{k+1}^{(\ell)}=P_{k}^{(\ell)}-\frac{P_{k}^{(\ell)} \varphi_{k}^{(\ell)} \varphi_{k}^{(\ell)^{T}} P_{k}^{(\ell)}}{1+\varphi_{k}^{(\ell) T} P_{k}^{(\ell)} \varphi_{k}^{(\ell)}}, \quad P_{0}^{(\ell)}=P_{0}^{(\ell)^{T}}>0,
\end{gathered}
$$

where $\hat{\theta}_{k}^{(\ell)}$ and $\varphi_{k}^{(\ell)}$ were defined in Section 2.2, and $e_{k}^{(\ell)}=y_{k}-\hat{y}_{k}^{(\ell)}=y_{k}-\hat{\theta}_{k}^{(\ell)^{T}} \varphi_{k}^{(\ell)}$ is the identification error corresponding to the $\ell$ th estimation model at the $k$ sample time instant.

Algorithm 5.4 (with dead zone). This algorithm is of least-squares type as well, but it incorporates a relative adaptation dead zone. The estimates are given by (2.12).

A comparison between the identification performances which can be gotten from those algorithms is discussed through simulations below.

5.4. Simulations. This section illustrates the performance of the multiestimation scheme for identifying the parameters of the transfer function associated with the current operation point of a nonlinear plant. The improvement in the identification by the addition of 
a relative adaptation dead zone in each algorithm which composes the multi-estimation scheme when the influence of the unmodeled dynamics is relevant will be numerically shown. A discrete stable nonlinear plant with three known modes is considered; namely, $p_{1}=0.5, p_{2}=0.25$, and $p_{3}=-0.75$. Therefore, the behavior of the plant at a certain operating point can be described by

$$
H(z)=\frac{k_{1,1}}{z-0.5}+\frac{k_{2,1}}{z-0.25}+\frac{k_{3,1}}{z+0.75}
$$

where $k_{1,1}, k_{2,1}$, and $k_{3,1}$ are unknown and they have to be estimated. The sampling time is $T_{s}=0.1 s$. At least one of the simple filters of the whole plant model (2.5) is suppressed in order to describe the various reduced-order estimated models of the plant for each possible operating point. In this way, six possible reduced-order nominal models can be obtained. In particular, a subset composed by only the three estimation models of second order is considered in the multiestimation scheme. Each one misses information about one of the simple plant modes. The three estimation models of two poles used for the scheme's implementation are

$$
\begin{gathered}
\hat{H}^{(1)}(z)=\frac{\hat{k}_{1,1}^{(1)}}{z-0.5}+\frac{\hat{k}_{2,1}^{(1)}}{z-0.25}, \quad \hat{H}^{(2)}(z)=\frac{\hat{k}_{1,1}^{(2)}}{z-0.5}+\frac{\hat{k}_{3,1}^{(2)}}{z+0.75}, \\
\hat{H}^{(3)}(z)=\frac{\hat{k}_{2,1}^{(3)}}{z-0.25}+\frac{\hat{k}_{3,1}^{(3)}}{z+0.75} .
\end{gathered}
$$

The gains of each simple partial fraction of each reduced-order model are estimated by using any of the estimation Algorithms 5.3 or 5.4. Two simulations are developed for each operating point parameterization of the true plant. The first one uses Algorithm 5.3 for all the estimators which compose the multiestimation scheme and the second one uses Algorithm 5.4, that is, a relative adaptation dead zone is included in each estimator. All the estimation algorithms are initialized with $\hat{k}_{j, 1}^{(\ell)}=1$ and $P_{0}^{(\ell)}=10^{15} I_{2}$, with $j, \ell \in$ $\{1,2,3\}$, where $I_{2}$ denotes the second-order identity matrix. The selection of the best reduced-order estimated model by the supervisor is performed after each ten samples, that is, at the sampling instants $k=l N_{r}$ for all integers $l>0$ with $N_{r}=10$. The forgetting factor which appears in the identification quality index $J_{k}^{(\ell)}$ of $(2.17)$, for each reducedorder model, is $\lambda=0.995$ and the weighting factor $\alpha$ is fixed to unity. The parameters of the relative adaptation dead zone included in Algorithm 5.4 are $\mu^{(\ell)}=1.1$ and $\sigma^{(\ell)}=$ 0.995 , for all $\ell \in\{1,2,3\}, \alpha_{0}^{(1)}=\alpha_{1}^{(1)}=0.2, \alpha_{0}^{(2)}=0.75, \alpha_{1}^{(2)}=1$, and $\alpha_{0}^{(3)}=\alpha_{1}^{(3)}=2$.

5.4.1. Simulation 1: comparison of the identification results with or without dead zone in the estimators. The input applied to the plant is $u_{k}=\sin (\pi k / 100)+0.25 \sin (\pi k / 25)$. The behavior of the plant subject to this input is given by (5.2) with the unknown values $k_{j, 1}^{(i)}=2$ for $i \in\{1,2,3\}$. The three estimation models are included in the multiestimation scheme in order to obtain a reduced-order estimation model for the plant at this operation point. Therefore, each estimation model estimates two parameters. Figure 5.1a shows the estimation model chosen by the supervisor during the simulation when 


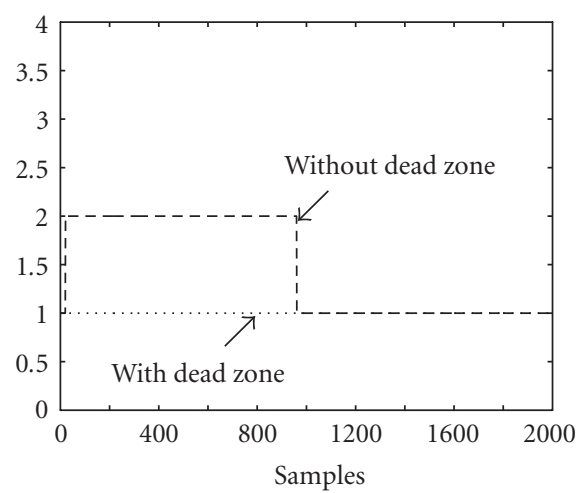

(a)

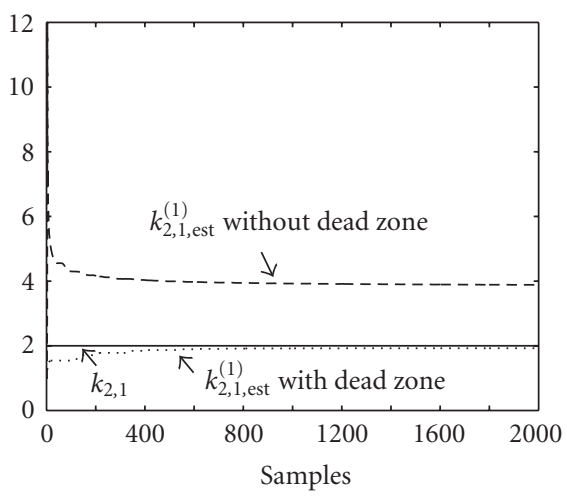

(c)

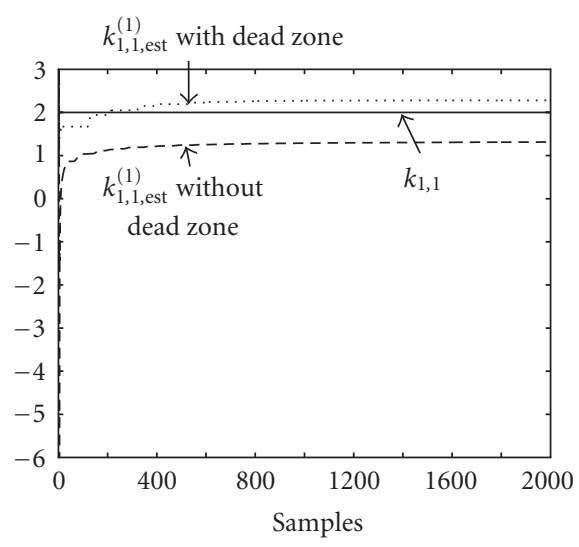

(b)

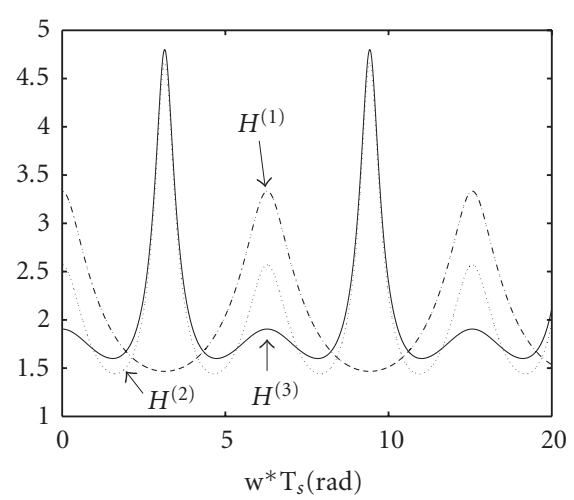

(d)

Figure 5.1. (a) Active reduced-order estimation model. (b) Identification of the parameter $k_{1,1}^{(1)}$. (c) Identification of the parameter $k_{2,1}^{(1)}$. (d) Amplitude Bode diagram of the three nominal estimation models.

Algorithm 5.3 is used in all estimators (dashed line) and when Algorithm 5.4, that is, Algorithm 5.3 with dead zone, is used in all estimators (dotted line). In any case the supervisor finally chooses the estimation model $\hat{H}^{(1)}(z)$ which turns out to be the best reduced-order estimation model for the true plant at this operation point. The true values $k_{1,1}$ and $k_{2,1}$ (solid lines) together with the evolution of the time-varying parameters $\hat{k}_{1,1}^{(1)}$ and $\hat{k}_{2,1}^{(1)}$ corresponding to the estimation model $H^{(1)}(z)$ are displayed, respectively, in Figures 5.1b and 5.1c when Algorithm 5.3 is used (dashed line) and when Algorithm 5.4 is used (dotted line).

The reason for the final choice of the estimation model $\hat{H}^{(1)}(z)$ by the supervisor is based in the frequency spectrum of the applied input. The input signal is the sum of two sinusoidal signals of frequencies $0.314 \mathrm{rad} / \mathrm{s}$ and $1.256 \mathrm{rad} / \mathrm{s}$. The amplitude Bode 
diagram is displayed in Figure 5.1d for each of the reduced-order estimation nominal models with $\hat{k}_{j, 1}^{(i)}=1$, for all $j, i \in\{1,2,3\}$ and all integers $k \geq 0$. Such a figure highlights the gain for the reduced-order models $\hat{H}^{(\ell)}(z)$ within the relevant frequencies which characterizes the applied input. Consequently, it is finally chosen by the switching rule of the supervisor as the best identification model since it gives the largest gain for the applied input. Figures 5.1b and 5.1c display the evolution of the parameters of the estimation model $H^{(1)}(z)$, which is finally chosen by the supervisor at each time interval. These estimates converge to constant values which do not coincide exactly with the corresponding true values of (5.2). The reason is that the estimation model is of a reduced-order type. Moreover, the parametrical error magnitude depends on the type of the identification algorithm. Figures 5.1b and 5.1c show that the identification is better if a relative adaptation dead zone is included in the estimation algorithms which compose the multiestimation scheme. That is, the identification is improved if the estimation algorithms include such a tool to deal with the unmodeled dynamics associated with the simple partial fraction not included in the estimation model selected by the supervisor. However, the inclusion of the relative adaptation dead zone only improves the identification of the reduced-order estimated model if the unmodeled dynamics contribution is higher than a certain threshold compared to the contribution to the output of the nominal part of the reduced-order model. Such a feature will be highlighted in the subsequent numerical example.

\subsubsection{Example 2: influence of the unmodeled dynamics in the identification performance.}

Figure 5.2 displays the results for the plant at an operation point (5.2) with the unknown parameters $k_{i, 2}=2$ for $i \in\{1,2\}$ and $k_{3,1}=0.1$ to be identified, under the same input signal of the previous numerical example.

In this case, the contribution of the unmodeled dynamics, which comes from the suppressed simple partial fraction in the activated estimation model by the supervisor (i.e., from $k_{3,1} /(z+0.75)$ during all the simulation), is small enough and the identification is worse if the dead zone is included in the estimation algorithms. These simulations illustrate that the identification performances of the multiestimation scheme, without dead zone or with dead zone in the estimators, depend on the magnitude of the unmodeled dynamics for the relevant input frequency spectrum. If the related contribution to the output is small enough, then the inclusion of a relative adaptation dead zone does not improve, in general, the identification performance under an arbitrary reference input signal. However, if the input frequency spectrum is close to the unmodeled dynamics frequency range, then the inclusion of a relative adaptation dead zone in each estimation algorithm is going to improve the identification performance. Then, an input whose frequency spectrum is far from the frequencies associated with the unmodeled dynamics will be suitable to obtain a good identification of the system parameters. The following figures show the recursive identification updating of the parameters of the reduced-order estimation model chosen by the supervisor at the end of the simulations, that is, the estimation model $\hat{H}^{(1)}(z)$, as a function of the parameter $k_{3,1}$ related to the unmodeled dynamics associated with such an estimation model. Note that the convergence of the estimates to the true values would be possible if the gain of the suppressed plant mode were zero and the estimation algorithm without dead zone were used in the multiestimation 


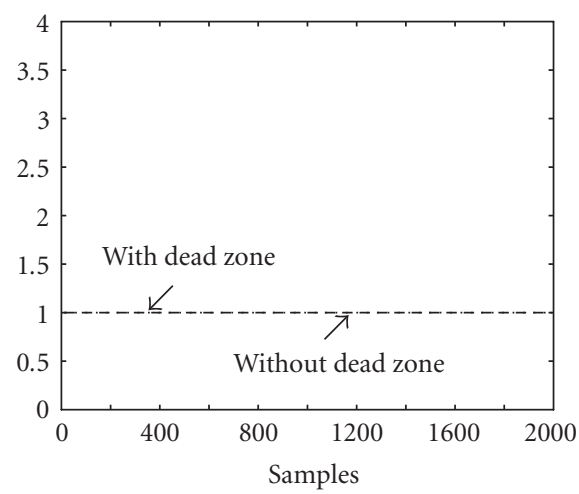

(a)

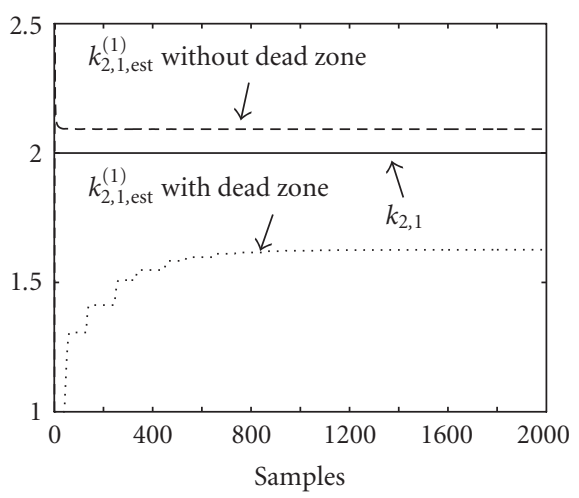

(c)

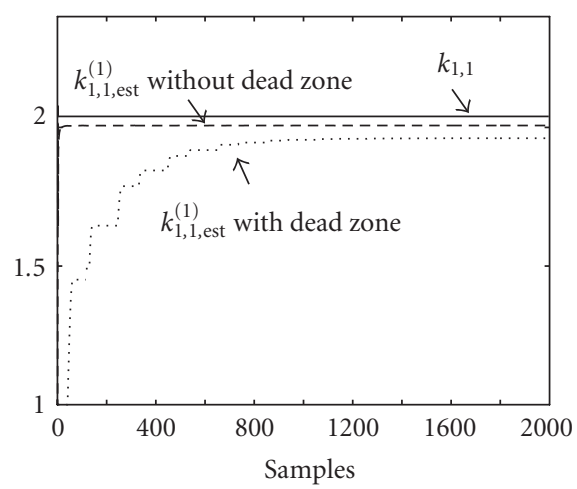

(b)

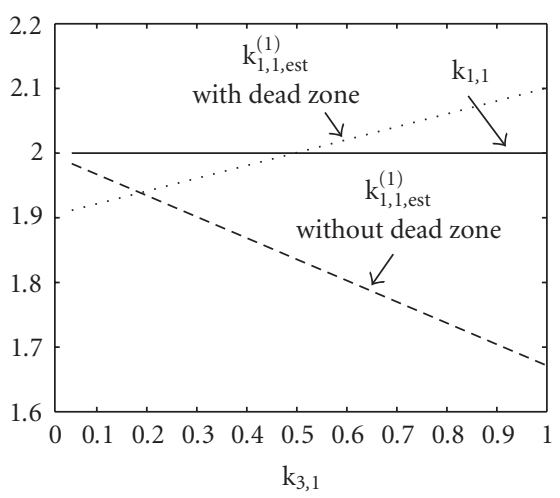

(d)

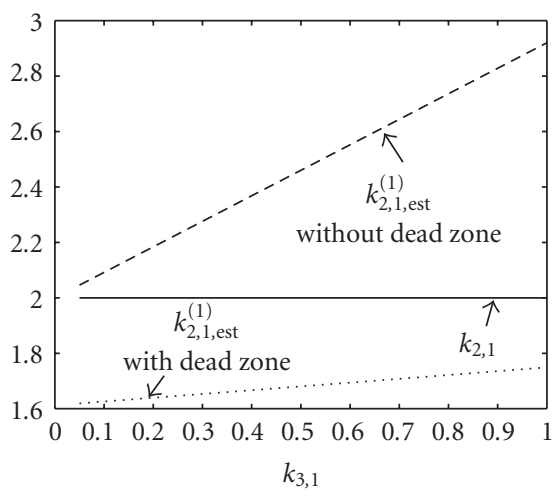

(e)

Figure 5.2. (a) Active reduced-order estimation model. (b) Identification of the parameter $k_{1,1}^{(1)}$. (c) Identification of the parameter $k_{2,1}^{(1)}$. (d) Identification of the parameter $k_{1,1}^{(1)}$ as a function of the unmodeled dynamics magnitude. (e) Identification of the parameter $k_{2,1}^{(1)}$ as a function of the unmodeled dynamics magnitude. 
scheme. The use of the multiestimation scheme is justified from the point of view that the gains of the plant modes are a priori unknown and then it is worthwhile that different estimation models are being updated simultaneously and a supervisor is selecting the estimation model which is the best approximation to the operation point of the true plant at each certain time interval.

5.4.3. Identification using a class of a swept-frequency cosine generator (chirp) wave input. In this section, the frequency of the signal input is periodically changed between two prefixed values in order to show how the most appropriate reduced-order model depends through time on the time-varying input frequency. During the first 2000 samples, the input signal may be described as follows:

$$
\begin{aligned}
& u_{k}=\cos \left(2 \pi(1+0.299 k) 10^{-3} k\right) \quad \text { if } 0 \leq k \leq 1000 \\
& u_{k}=u_{2000-k} \quad \text { if } 1000<k \leq 2000
\end{aligned}
$$

It means that the frequency varies from $0.01 \mathrm{~Hz}$ until $3 \mathrm{~Hz}$ during the first 1000 samples and then it decreases until its initial value. Then, the input signal is repeated with the same pattern four times until the 8000th sample, maintaining the last frequency fixed in the following samples. The same transfer function (5.2) is used with $k_{i, 1}=2$ for $i \in$ $\{1,2,3\}$. Figure 5.3a shows the evolution of the estimator with the best estimation index. The simulation shows the results using the algorithm with and without the relative dead zone in the identification. We can see that the system does not select any estimator as the best one all the time while the frequency is being changed. This fact shows that there is, in general, no appropriate reduced-order model for the whole range of frequencies and the usefulness of each one is related to a determinate range in the frequency spectra. This proves the necessity of including the whole set of reduced-order models with the aim of covering the largest possible range in the frequency spectra. The estimated values are shown in Figures 5.3b, 5.3c, and 5.3d as well. Figure 5.3d, where the multiestimation scheme is used, shows the estimated value of the $k_{1,1}$ gain according to the best estimator at that moment. That's why there are some discontinuities in the figure when the system switches to another estimator. However, each estimator does not take into account all the unknown gains; it only estimates a subgroup of them so that the last estimated value is maintained until another estimator, which includes the estimation of that gain, is found to have the best performance index. Figures $5.3 \mathrm{~b}$ and $5.3 \mathrm{c}$ show the estimation of the same gain if the estimator related to the first or second reduced-order model, respectively, is maintained during the whole simulation.

5.5. Remarks. A multiestimation scheme has been presented to obtain a reduced-order estimated model for a (possibly nonlinear) stable real plant at the current operating point. Each of the nominal models to be estimated suppresses at least one of the plant modes. The estimation algorithms within the multiestimation scheme run in parallel and estimate the gain associated with each mode included in its corresponding nominal model. The simulation results corroborate the achievable performance when using reduced-order estimation models of the true plant at the current operation point. The improvement in the identification performance is apparent with the inclusion of 


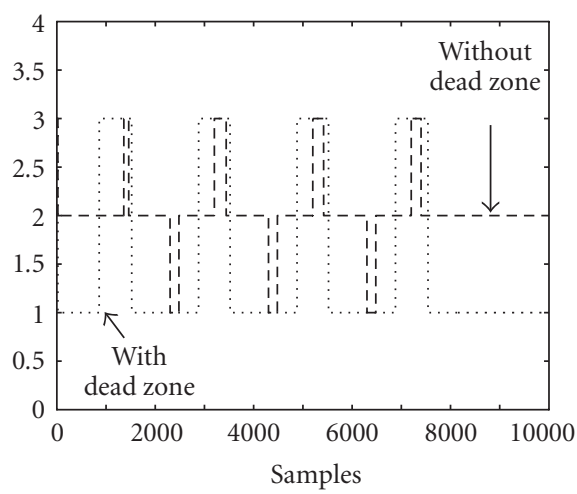

(a)

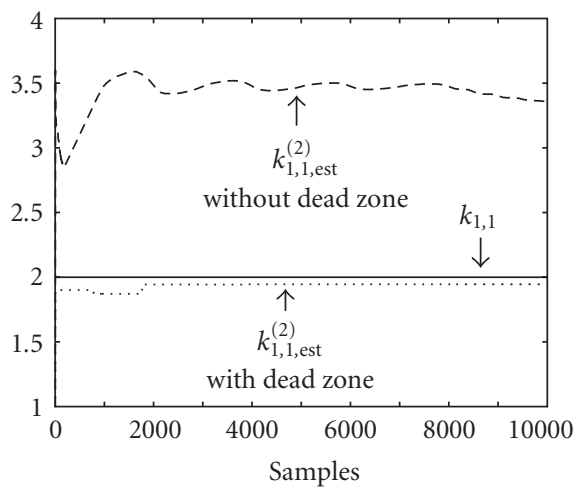

(c)

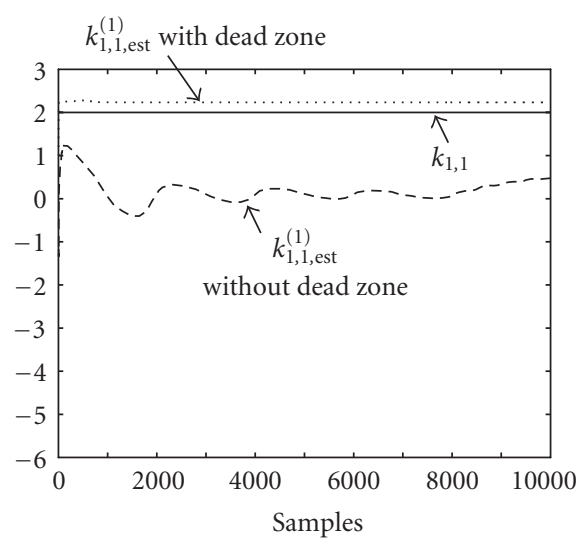

(b)

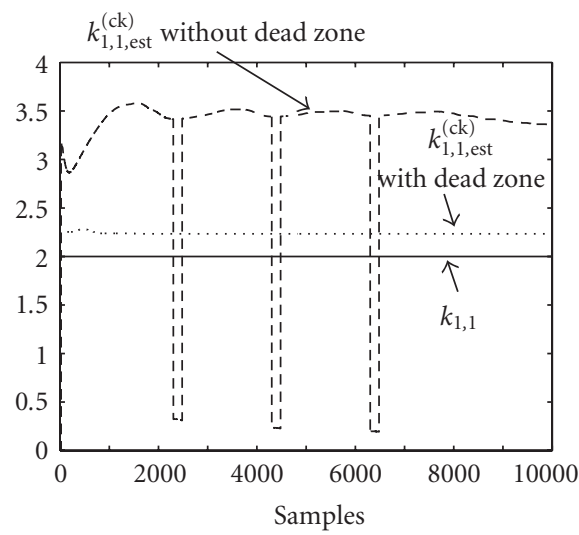

(d)

Figure 5.3. (a) Active estimator. (b) Estimated value of $k_{1,1}^{(1)}$. (c) Estimated value of $k_{1,1}^{(2)}$ (d) Estimated value of $k_{1,1}^{\left(c_{k}\right)}$ using the multiestimation scheme.

a relative adaptation dead zone if the contribution of the unmodeled dynamics to the system output is relevant or if the spectrum of the plant input is close to the frequency range of the unmodeled dynamics. The reason is that some of the estimators do not operate efficiently for such frequencies. A good reduced-order model of the true plant can be obtained subject to an input whose frequency spectrum is far from the frequencies associated to the unmodeled dynamics.

\section{Conclusions}

In this work, a multiestimation scheme for discrete adaptive control has been presented. The various estimation schemes are, in general, associated with parameterized reducedorder plant models plus extra unmodeled dynamics and possess a relative adaptation 
dead zone. Such a dead zone freezes the estimation process when the absolute value of the identification error is smaller than a known absolute upper bound of the contribution of the unmodeled dynamics to the output. It is only requested that at least one of the reduced-order models possess stable unmodeled dynamics which is also a main theoretical contribution of this paper related to the previous literature on the subject. A supervisor with two hierarchized supervision levels selects on-line the appropriate estimation model which gives the best identification performance to update the adaptive controller parameterization via switches through time. Each estimation model generates a poleplacement-based parameterization of the adaptive controller with only one of them being in fact in operation at each time interval. The selection of the estimated model is made automatically by minimizing an identification performance index while respecting a minimum residence time between consecutive switches at each estimator/controller parameterization so as to guarantee closed-loop stability. It is shown that the most recent input frequency range is very relevant in the online current estimator to be selected to parameterize the adaptive controller. It is due to the fact that the effective loop-gain highly depends on the input frequency spectrum because of the filtering effect of the plant and then on its reduced-order model being more relevant to the input band frequency. The scheme has been proved to guarantee the closed-loop stability if the switchings between the various estimators are subject to a minimum dwell time, which can be estimated either from a priori knowledge or through an ad hoc on-line computation algorithm. It has been proved that a judicious choice of the switching rule allows the designer to obtain relevant improvements in the identification and transient closed-loop control performances in the case when the choice of a fixed constant value yields a worse transient behavior. It can be concluded that the multiestimation scheme is an effective option for the development of high-performance transient response adaptive controllers, especially for plants that are highly uncertain (i.e., the compact set $\mathbf{D}$ where the real plant parameter vector belongs is very large). For such plants, a significant adaptation transient response improvement can be achieved in two ways, that is, the output overshoot peaks and the accumulated deviations from the desired output are smaller in this case than in the conventional adaptive control basic scheme. The scheme also achieves an acceptable performance when applied to plants whose parameters change abruptly with time. In those cases, the multiestimation scheme gives a better performance than the conventional adaptive configuration not only because of the switching but also because of the supervision. The proposed scheme reveals to be promising for the adaptive control of time-varying discrete systems as well. The scheme has also been adapted to the open-loop identification problem without using any controller to more clearly elucidate the relevance of the various reduced-order models to the parameter estimation depending on the frequency spectrum of a prefixed plant test input. Simulations have corroborated through numerical examples all the expected results from the previous theoretically addressed analysis.

\section{Appendices}

\section{A. Proof of Theorem 3.1.}

In this proof the superscripts denoting each estimation scheme $\ell \in N_{e}$ in the multiestimation scheme are deleted in the notation for simplicity so that no confusion arises. For each 
$\ell$ th estimation scheme, define the following positive definite sequence $V_{k}=\tilde{\theta}_{k}^{T} P_{k}^{-1} \tilde{\theta}_{k}$, with the associated nominal parametrical error $\tilde{\theta}_{k}=\theta-\hat{\theta}_{k}$ and covariance matrix $P_{k}$. Its one-step increment is

$$
\begin{aligned}
\Delta V_{k} & =V_{k+1}-V_{k} \\
& =\left(\tilde{\theta}_{k}^{T}-\phi_{k} \varphi_{k}^{T} P_{k} e_{k}\right)\left(P_{k}^{-1}+\frac{s_{k} \varphi_{k} \varphi_{k}^{T}}{1+\left(1-s_{k}\right) \varphi_{k}^{T} P_{k} \varphi_{k}}\right)\left(\tilde{\theta}_{k}-\phi_{k} P_{k} \varphi_{k} e_{k}\right)-\tilde{\theta}_{k}^{T} P_{k} \tilde{\theta}_{k}
\end{aligned}
$$

since $P_{k+1}^{-1}=\left(P_{k}^{-1}+s_{k} \varphi_{k} \varphi_{k}^{T} /\left(1+\left(1-s_{k}\right) \varphi_{k}^{T} P_{k} \varphi_{k}\right)\right)$ by using the matrix inversion lemma in (2.14a), where $\phi_{k}=s_{k} /\left(1+\varphi_{k}^{T} P_{k} \varphi_{k}\right)$. Equation (A.1) may be rewritten as

$$
\begin{aligned}
\Delta V_{k}= & -\frac{s_{k}}{\left(1+\varphi_{k}^{T} P_{k} \varphi_{k}\right)^{2}\left(1+\left(1-s_{k}\right) \varphi_{k}^{T} P_{k} \varphi_{k}\right)} \\
& \times\left(2\left(\tilde{\theta}_{k}^{T} \varphi_{k}\right) e_{k}-\left(\tilde{\theta}_{k}^{T} \varphi_{k}\right)^{2}+\varphi_{k}^{T} P_{k} \varphi_{k}\left(4\left(\tilde{\theta}_{k}^{T} \varphi_{k}\right) e_{k}-2\left(\tilde{\theta}_{k}^{T} \varphi_{k}\right)^{2}-s_{k} e_{k}^{2}\right)\right. \\
& \left.\quad+\left(\varphi_{k}^{T} P_{k} \varphi_{k}\right)^{2}\left(-\left(\tilde{\theta}_{k}^{T} \varphi_{k}\right)^{2}+2\left(\tilde{\theta}_{k}^{T} \varphi_{k}\right) e_{k}-s_{k} e_{k}^{2}\right)\right) .
\end{aligned}
$$

Now, replacing $\tilde{\theta}_{k}^{T} \varphi_{k}=e_{k}-\eta_{k}$ in the previous equation,

$$
\begin{aligned}
\Delta V_{k}= & -\frac{s_{k}}{\left(1+\varphi_{k}^{T} P_{k} \varphi_{k}\right)^{2}\left(1+\left(1-s_{k}\right) \varphi_{k}^{T} P_{k} \varphi_{k}\right)} \\
& \times\left(e_{k}^{2}-\eta_{k}^{2}+\varphi_{k}^{T} P_{k} \varphi_{k}\left(\left(2-s_{k}\right) e_{k}^{2}-2 \eta_{k}^{2}\right)+\left(\varphi_{k}^{T} P_{k} \varphi_{k}\right)^{2}\left(\left(1-s_{k}\right) e_{k}^{2}-\eta_{k}^{2}\right)\right) \\
= & -\frac{s_{k}}{\left(1+\varphi_{k}^{T} P_{k} \varphi_{k}\right)\left(1+\left(1-s_{k}\right) \varphi_{k}^{T} P_{k} \varphi_{k}\right)}\left(\varphi_{k}^{T} P_{k} \varphi_{k}\left(e_{k}^{2}\left(1-s_{k}\right)-\eta_{k}^{2}\right)+e_{k}^{2}-\eta_{k}^{2}\right) .
\end{aligned}
$$

When the algorithm is not frozen, $\left|e_{k}\right|>\mu \bar{\eta}_{k}\left(\right.$ if not $\left.\Delta V_{k}=0\right)$ :

$$
\begin{aligned}
\Delta V_{k} & =-\frac{s_{k}}{\left(1+\varphi_{k}^{T} P_{k} \varphi_{k}\right)\left(1+\left(1-s_{k}\right) \varphi_{k}^{T} P_{k} \varphi_{k}\right)}\left(\varphi_{k}^{T} P_{k} \varphi_{k}\left(\mu \bar{\eta}_{k}\left|e_{k}\right|-\eta_{k}^{2}\right)+e_{k}^{2}-\eta_{k}^{2}\right) \\
& \leq-\frac{s_{k}}{\left(1+\varphi_{k}^{T} P_{k} \varphi_{k}\right)\left(1+\left(1-s_{k}\right) \varphi_{k}^{T} P_{k} \varphi_{k}\right)}\left(\varphi_{k}^{T} P_{k} \varphi_{k}\left(\mu^{2} \bar{\eta}_{k}^{2}-\eta_{k}^{2}\right)+\mu^{2} \bar{\eta}_{k}^{2}-\eta_{k}^{2}\right) \\
& =-\frac{s_{k}}{\left(1+\left(1-s_{k}\right) \varphi_{k}^{T} P_{k} \varphi_{k}\right)}\left(\mu^{2} \bar{\eta}_{k}^{2}-\eta_{k}^{2}\right) \leq 0,
\end{aligned}
$$

where $(2.14 \mathrm{~b})$ has been used. Thus, $\Delta V_{k} \leq 0$ and $V_{k}=\tilde{\theta}_{k}^{T} P_{k}^{-1} \tilde{\theta}_{k} \leq V_{0}<\infty$, for any bounded $\hat{\theta}_{0}$ and $P_{0}^{-1}$ while it tends to a bounded nonnegative real constant asymptotically as $k \rightarrow \infty$ since it is uniformly bounded, monotonically decreasing, and nonnegative for all integers $k \geq 0$. Also, $\infty>V_{0}-V_{\infty}=\sum_{k=0}^{\infty}\left|\Delta V_{k}\right|<\infty$ and $\lim _{k \rightarrow \infty}\left|\Delta V_{k}\right|=0$. Moreover, 
$\infty>V_{k} \geq 1 /\left(\lambda_{\max }\left(P_{0}\right)\right)\left\|\tilde{\theta}_{k}\right\|^{2}$ implies $\left\|\tilde{\theta}_{k}\right\|<\infty$ and $\left\|\hat{\theta}_{k}\right\|<\infty$. Properties 1 and 2 follow directly from the previous equation and (A.3). Property 3 is proved using property 2 ,

$$
\begin{aligned}
\infty & >\sum_{k=0}^{\infty} \frac{s_{k} \varphi_{k}^{T} P_{k} \varphi_{k}\left(e_{k}^{2}\left(1-s_{k}\right)-\eta_{k}^{2}\right)}{\left(1+\varphi_{k}^{T} P_{k} \varphi_{k}\right)\left(1+\left(1-s_{k}\right) \varphi_{k}^{T} P_{k} \varphi_{k}\right)} \\
& =\sum_{k=0}^{\infty} \frac{s_{k} \varphi_{k}^{T} P_{k} \varphi_{k}\left(\mu \bar{\eta}_{k}\left|e_{k}\right|-\eta_{k}^{2}\right)}{\left(1+\varphi_{k}^{T} P_{k} \varphi_{k}\right)\left(1+\left(1-s_{k}\right) \varphi_{k}^{T} P_{k} \varphi_{k}\right)} \\
& \geq \sum_{k=0}^{\infty} \frac{s_{k} \varphi_{k}^{T} P_{k} \varphi_{k} \eta_{k}^{2}\left(\mu^{2}-1\right)}{\left(1+\varphi_{k}^{T} P_{k} \varphi_{k}\right)\left(1+\left(1-s_{k}\right) \varphi_{k}^{T} P_{k} \varphi_{k}\right)} \\
& \geq 0 \Longrightarrow \sum_{k=0}^{\infty} \frac{s_{k} \varphi_{k}^{T} P_{k} \varphi_{k} \eta_{k}^{2}}{\left(1+\varphi_{k}^{T} P_{k} \varphi_{k}\right)\left(1+\left(1-s_{k}\right) \varphi_{k}^{T} P_{k} \varphi_{k}\right)}<\infty,
\end{aligned}
$$

and the fact that the following sum of sequences is bounded as well:

$$
\begin{aligned}
\infty & >\sum_{k=0}^{\infty}\left|\Delta V_{k}\right|+\sum_{k=0}^{\infty} \frac{s_{k}^{2} \varphi_{k}^{T} P_{k} \varphi_{k} \eta_{k}^{2}}{\left(1+\varphi_{k}^{T} P_{k} \varphi_{k}\right)\left(1+\left(1-s_{k}\right) \varphi_{k}^{T} P_{k} \varphi_{k}\right)} \\
& =\sum_{k=0}^{\infty} \frac{s_{k}\left(e_{k}^{2}-\eta_{k}^{2}\right)}{1+\varphi_{k}^{T} P_{k} \varphi_{k}} \geq \sum_{k=0}^{\infty} \frac{s_{k} e_{k}^{2}\left(1-\mu^{-2}\right)}{1+\varphi_{k}^{T} P_{k} \varphi_{k}} \Longrightarrow \sum_{k=0}^{\infty} \frac{s_{k} e_{k}^{2}}{1+\varphi_{k}^{T} P_{k} \varphi_{k}}<\infty,
\end{aligned}
$$

then property 3 is proved as well.

From property $3, \phi_{k} e_{k}^{2} \rightarrow 0$ as $k \rightarrow \infty$ follows directly. As a result, either $\phi_{k} \rightarrow 0$ or $e_{k}^{2} \rightarrow 0$. However, if $e_{k}^{2} \rightarrow 0$ as $k \rightarrow \infty$, then $\phi_{k} \rightarrow 0$ as $k \rightarrow \infty$ from (2.14b). Thus, $\phi_{k} \rightarrow 0$ as $k \rightarrow \infty$. On the other hand, if the regressor is bounded for each estimator, the above asymptotic properties are achieved by replacing $\phi_{k}$ with $s_{k}$, and property 4 is proved. The last property is proved in the following way:

$$
\begin{aligned}
\sum_{k=0}^{\infty}\left\|\hat{\theta}_{k+1}-\hat{\theta}_{k}\right\|^{2} & =\sum_{k=0}^{\infty}\left\|\frac{s_{k} P_{k} \varphi_{k} e_{k}}{1+\varphi_{k}^{T} P_{k} \varphi_{k}}\right\|^{2} \\
& \leq \sum_{k=0}^{\infty} \frac{\lambda_{\max }\left(P_{0}\right) s_{k}^{2} e_{k}^{2}}{1+\varphi_{k}^{T} P_{k} \varphi_{k}}<\infty \Longrightarrow \lim _{k \rightarrow \infty}\left\|\hat{\theta}_{k+1}-\hat{\theta}_{k}\right\|=0 .
\end{aligned}
$$

Moreover, since $\left\|\hat{\theta}_{k+m}-\hat{\theta}_{k}\right\|^{2} \leq m\left(\left\|\hat{\theta}_{k+m}-\hat{\theta}_{k+m-1}\right\|^{2}+\left\|\hat{\theta}_{k+m-1}-\hat{\theta}_{k+m-2}\right\|^{2}+\cdots+\right.$ $\left\|\hat{\theta}_{k+1}-\hat{\theta}_{k}\right\|^{2}$ ), then $\lim _{k \rightarrow \infty}\left\|\hat{\theta}_{k+m}-\hat{\theta}_{k}\right\|=0$ for $m>0$ (finite) and property 5 is proved.

\section{B. Intermediate auxiliary results and proof of Theorem 3.4}

The following intermediate result will be used in the proof of Theorem 3.4.

Lemma B.1. There exists a sufficiently large finite integer $k_{0} \geq 0$ such that

$$
\operatorname{Max}_{\ell \in N_{e}}\left(\left|e_{k}^{(\ell)}\right|\right) \leq \alpha_{1}^{\prime} \operatorname{Sup}_{0 \leq j \leq k}\left(\sigma^{k-j} \| \varphi_{j}||\right)+\alpha_{0} \quad \forall k \geq k_{0}, \ell \in N_{e s}
$$


with

$$
\begin{gathered}
\alpha_{1}^{\prime}=\operatorname{Max}_{\ell \in N_{e s}}\left(\alpha_{1}^{(\ell)}+\left\|\tilde{\theta}_{\infty}^{(\ell)}\right\|\right) \geq 0, \quad \alpha_{0}=\operatorname{Max}_{\ell \in N_{e s}}\left(\alpha_{0}^{(\ell)}\right) \geq 0, \\
\sigma=\operatorname{Max}_{\ell \in N_{e s}}\left(\sigma^{(\ell)}\right) \in(0,1),
\end{gathered}
$$

where $\varphi_{j}$ denotes the highest dimensional regressor $\varphi_{j}^{(\ell)}$ in the multiestimation scheme.

Proof of Lemma B.1. Note from (2.11) that

$$
\begin{aligned}
\left|e_{k}^{(\ell)}\right| & \leq\left\|\varphi_{k}^{(\ell)}\right\| \cdot\left\|\tilde{\theta}_{k}^{(\ell)}\right\|+\bar{\eta}_{k}^{(\ell)} \\
& \leq\left\|\tilde{\theta}_{k}^{(\ell)}\right\| \sup _{0 \leq j \leq k}\left(\sigma^{(\ell) k-j}\left\|\varphi_{k}^{(\ell)}\right\|\right)+\alpha_{1}^{(\ell)} \sup _{0 \leq j \leq k}\left(\sigma^{(\ell) k-j}\left\|\varphi_{k}^{(\ell)}\right\|\right)+\alpha_{0}^{(\ell)} \\
& =\left(\left\|\tilde{\theta}_{k}^{(\ell)}\right\|+\alpha_{1}^{(\ell)}\right) \sup _{0 \leq j \leq k}\left(\sigma^{(\ell) k-j}\left\|\varphi_{k}^{(\ell)}\right\|\right)+\alpha_{0}^{(\ell)} .
\end{aligned}
$$

Proof of Theorem 3.4. As $G_{k}^{(\ell)}$ has bounded entries with its eigenvalues in $|z|<1$ for all $k \geq 0$ and satisfies Assumption 3.2, there exist real constants $K \geq 1$ (norm-dependent) and $\rho \in\left(\rho_{0}, 1\right)$ such that $\left\|\prod_{k=k_{0}+1}^{k_{0}+\ell+1} G_{k}^{\left(c_{k}\right)}\right\| \leq K \rho^{\ell}$ provided that the residence time is sufficiently large (see Lemma 3.3(ii)). Now, from (3.1), Assumptions 2.2 (3.1), and Lemma B.1, one gets, since $v_{k}$ is uniformly bounded and $\left\|x_{k}\right\| \geq\left\|\varphi_{k}^{(\ell)}\right\|$ for all $\ell \in N_{e}$ and all $k \geq k_{0}>0$ and sufficiently large, that $\left\|x_{k+N}\right\|$ is either bounded (and the proof is complete) or arbitrary large on an interval $\left(k_{0}, k_{0}+\ell T_{r}\right]$. Since at least one estimation scheme $i^{*}$ has stable unmodeled dynamics of known convergence abscissa $0<\sigma^{\left(i^{*}\right)} \leq \sigma<1$, then there exists $k \in\left(k_{0}, k_{0}+\ell N_{r}\right)$ such that

$$
\operatorname{Sup}_{0 \leq \ell \leq N}\left\|x_{k+\ell}\right\| \leq K\left\{\rho^{N}\left\|x_{k}\right\|+\frac{1}{1-\rho}\left[\alpha_{1}^{\prime} \operatorname{Sup}_{0 \leq \ell \leq k+N}\left(\left\|x_{\ell}\right\|\right)+\alpha_{0}\right]+K_{1}^{\prime}\right\} .
$$

Thus the sequence $\left\{\left\|x_{k}\right\| ; k \geq k_{0}\right\}$ cannot diverge if $\alpha_{1}^{\prime}<(1-\rho) / K$ (i.e., it is sufficiently small) since this implies that

$$
\operatorname{Sup}_{0 \leq \ell \leq N}\left(\left\|x_{\ell}\right\|\right) \leq\left(1-\frac{K \alpha_{1}^{\prime}}{1-\rho}\right)^{-1}\left(K \rho^{N}\left\|x_{0}\right\|+K^{\prime}\right)<\infty,
$$

for some constants $K>0$ and $K^{\prime}>0$.

If the transfer function describing the unmodeled dynamics is strictly proper, then the above constraints may be relaxed to $\alpha_{1}^{\prime} \leq(1-\rho) / k \sigma$ since Lemma B.1 may be rewritten as $\operatorname{Max}_{\ell \in N_{e}}\left(\left|e_{k}^{(\ell)}\right|\right) \leq \alpha_{1}^{\prime} \sigma \operatorname{Sup}_{0 \leq j \leq k-1}\left(\left\|\varphi_{j}\right\|\right)+\alpha_{0}$ in this case.

\section{Acknowledgment}

The authors are very grateful to MCYT for its partial support through Grant 2003-00164 and to the research groups 9/upv 00I06.I06-15263/2003 and 1/upv/ehu 00I06-00I06-EB $8235 / 2000$. 


\section{References}

[1] S. Alonso-Quesada and M. De la Sen, Robust adaptive control with multiple estimation models for stabilization of a class of non-inversely stable time-varying plants, Asian Journal of Control 6 (2004), no. 1, 59-73.

[2] K. J. Åström and B. Wittenmark, Computer Controlled Systems: Theory and Design, 2nd ed., Prentice-Hall, New Jersey, 1990.

[3] A. Bilbao-Guillerna, M. De la Sen, S. Alonso-Quesada, and A. Ibeas, A multiestimation adaptive control scheme which incorporates model reduction issues, 2nd WSEAS International Conference on Electronics, Control and Signal Processing (ICECS '03), Singapore, December 2003.

[4]_, Artificial intelligent tools for discrete multiestimation adaptive control scheme with model reduction issues, The IASTED International conference on Artificial Intelligence and Applications (AIA '04), Austria, February 2004.

[5] P. M. T. Broersen, A comparison of transfer function estimators, IEEE Trans. Instrumentation and Measurement 44 (1995), no. 3, 657-661.

[6] M. H. Chang and E. J. Davison, Adaptive switching control of LTI MIMO systems using a family of controllers approach, Automatica J. IFAC 35 (1999), no. 3, 453-465.

[7] Z. Chen, Y. Hayakawa, and S. Fujii, On the stabilizability of plants with variable operating conditions, Transactions of the Society of Instrument and Control Engineers 30 (1994), no. 9, 999-1004.

[8] M. De la Sen, S. Alonso-Quesada, A. Bilbao-Guillerna, and A. J. Garrido, A supervised multiestimation scheme for discrete adaptive control which guarantees robust closed-loop stability, Int. Math. J. 3 (2003), no. 9, 907-937.

[9] G. Feng, Analysis of a new algorithm for continuous-time robust adaptive control, IEEE Trans. Automat. Control 44 (1999), no. 9, 1764-1768.

[10] J. Frolik, M. Abdelrahman, and P. Kandasamy, A confidence-based approach to the selfvalidation, fusion and reconstruction of quasi-redundant sensor data, IEEE Trans. Instrumentation and Measurement 50 (2001), no. 6, 1761-1769.

[11] J. Hocherman-Frommer, S. R. Kulkarni, and P. J. Ramadge, Controller switching based on output prediction errors, IEEE Trans. Automat. Control 43 (1998), no. 5, 596-607.

[12] G. Kreisselmeier, Adaptive control of a class of slowly time-varying plants, Systems and Control Letters 8 (1986), no. 2, 97-103.

[13] S. R. Kulkarni and P. J. Ramadge, Model and controller selection policies based on output prediction errors, IEEE Trans. Automat. Control 41 (1996), no. 11, 1594-1604.

[14] B. Mårtensson, The order of any stabilizing regulator is sufficient a priori information for adaptive stabilization, Systems Control Lett. 6 (1985), no. 2, 87-91.

[15] R. H. Middleton, G. C. Goodwin, D. J. Hill, and D. Q. Mayne, Design issues in adaptive control, IEEE Trans. Automat. Control 33 (1988), no. 1, 50-58.

[16] A. S. Morse, Supervisory control of families of linear set-point controllers. I. Exact matching, IEEE Trans. Automat. Control 41 (1996), no. 10, 1413-1431.

[17] A. S. Morse, D. Q. Mayne, and G. C. Goodwin, Applications of hysteresis switching in parameter adaptive control, IEEE Trans. Automat. Control 37 (1992), no. 9, 1343-1354.

[18] E. Mosca and T. Agnoloni, Inference of candidate loop performance and data filtering for switching supervisory control, Automatica J. IFAC 37 (2001), no. 4, 527-534.

[19] K. S. Narendra and J. Balakrishnan, Improving transient response of adaptive control systems using multiple models and switching, IEEE Trans. Automat. Control 39 (1994), no. 9, 18611866.

[20] Adaptive control using multiple models, IEEE Trans. Automat. Control 42 (1997), no. 2, 171-187. 
[21] M. E. Stieber, E. M. Petriu, and G. Vukovich, Systematic design of instrumentation architecture for control of mechanical systems, IEEE Trans. Instrumentation and Measurement 45 (1996), no. 2, 406-412.

[22] S. R. Weller and G. C. Goodwin, Hysteresis switching adaptive control of linear multivariable systems, IEEE Trans. Automat. Control 39 (1994), no. 7, 1360-1375.

[23] E. Yashchin, Change-point models in industrial applications, Nonlinear Anal. 30 (1997), no. 7 , 3997-4006.

A. Bilbao-Guillerna: Instituto de Investigación y Desarrollo de Procesos, Facultad de Ciencia y Tecnología, Universidad del País Vasco, Campus de Leioa (Bizkaia), Apartado 644, 48080 Bilbao, Spain E-mail addresses: webbigua@ehu.es; bilgui@wanadoo.es

M. De la Sen: Instituto de Investigación y Desarrollo de Procesos, Facultad de Ciencia y Tecnología, Universidad del País Vasco, Campus de Leioa (Bizkaia), Apartado 644, 48080 Bilbao, Spain

E-mail address: msen@we.lc.ehu.es

A. Ibeas: Instituto de Investigación y Desarrollo de Procesos, Facultad de Ciencia y Tecnología, Universidad del País Vasco, Campus de Leioa (Bizkaia), Apartado 644, 48080 Bilbao, Spain

E-mail address: iebibhea@ehu.es

S. Alonso-Quesada: Instituto de Investigación y Desarrollo de Procesos, Facultad de Ciencia y Tecnología, Universidad del País Vasco, Campus de Leioa (Bizkaia), Apartado 644, 48080 Bilbao, Spain

E-mail address: santi@we.lc.ehu.es 


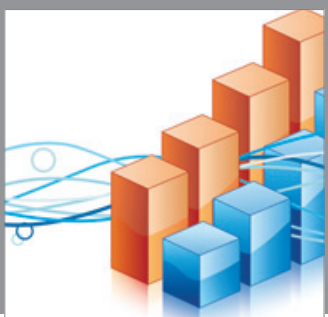

Advances in

Operations Research

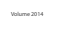

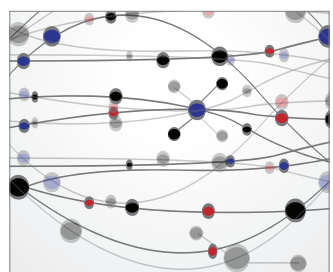

\section{The Scientific} World Journal
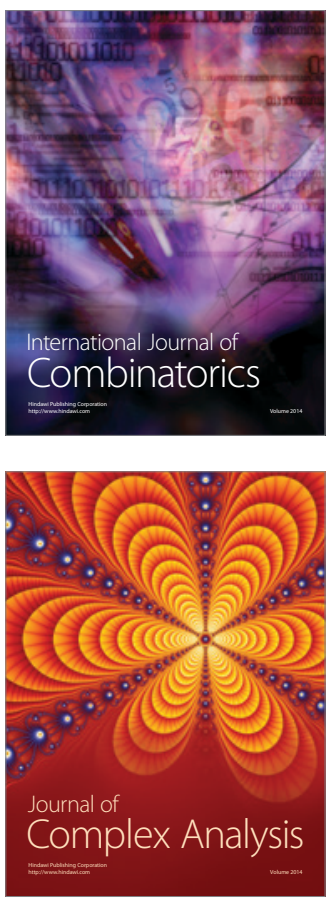

International Journal of

Mathematics and

Mathematical

Sciences
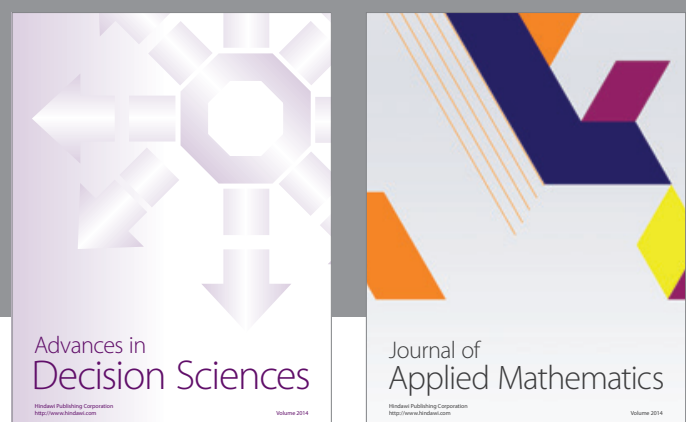

Journal of

Applied Mathematics
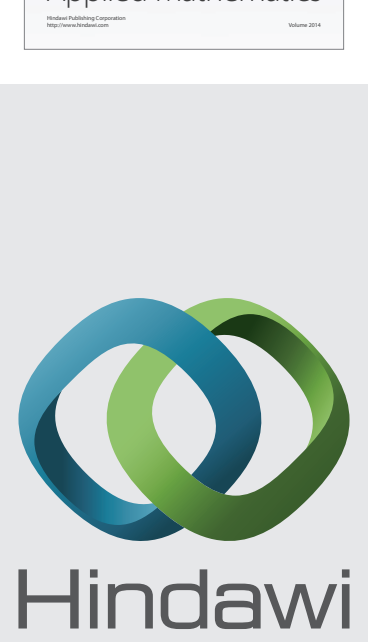

Submit your manuscripts at http://www.hindawi.com
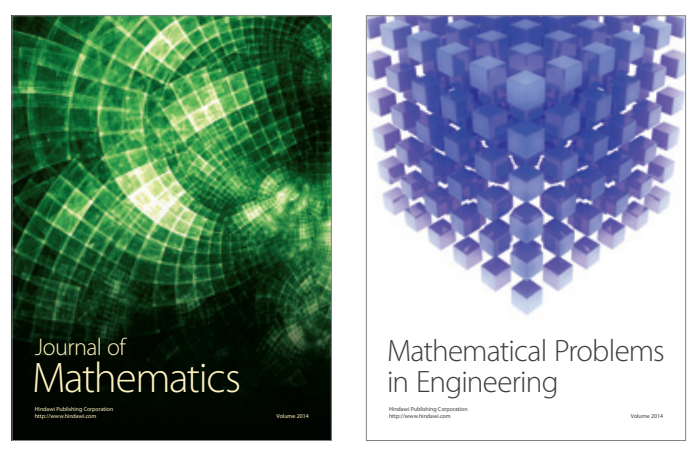

Mathematical Problems in Engineering
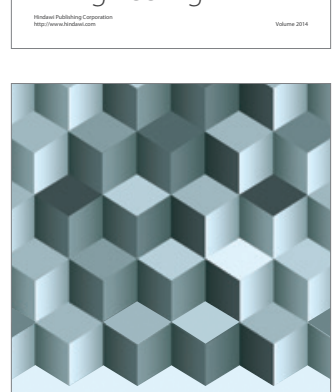

Journal of

Function Spaces
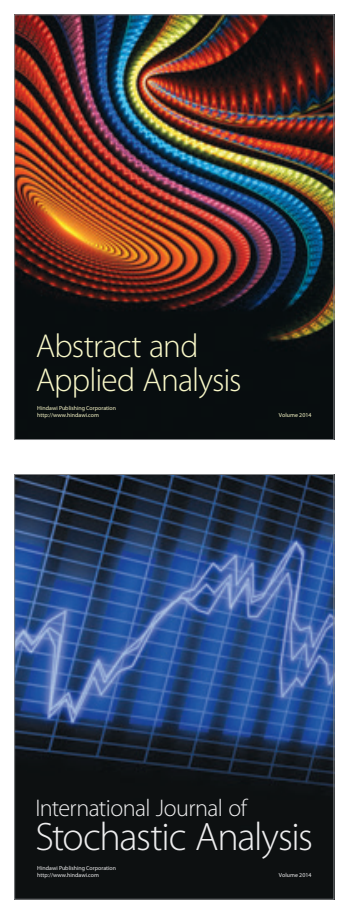

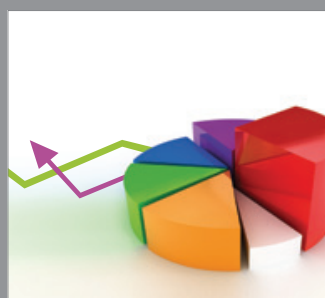

ournal of

Probability and Statistics

Promensencen
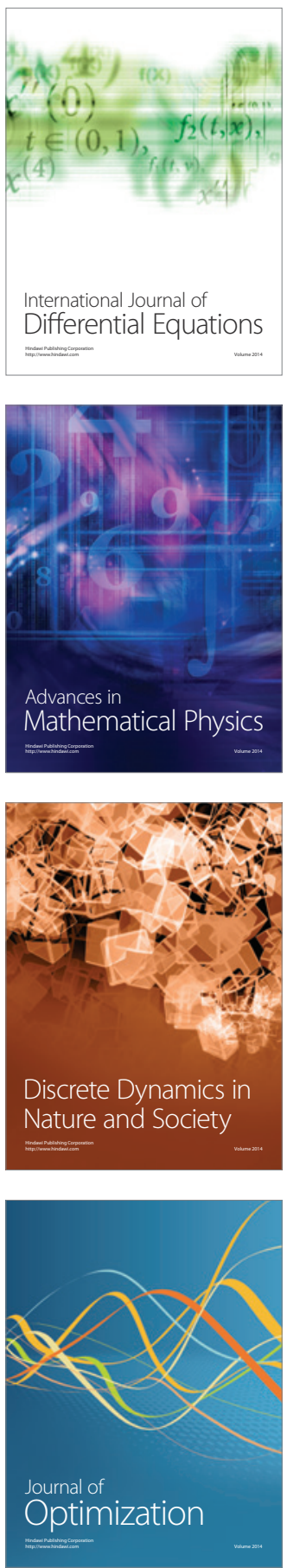